\title{
Quantum stochastic differential equations for boson and fermion systems - Method of Non-Equilibrium Thermo Field Dynamics
}

\author{
A.E. Kobryn ${ }^{1}$, T. Hayashi and T. Arimitsu* \\ Institute of Physics, University of Tsukuba, Ibaraki 305-8571, Japan
}

\begin{abstract}
A unified canonical operator formalism for quantum stochastic differential equations, including the quantum stochastic Liouville equation and the quantum Langevin equation both of the Itô and the Stratonovich types, is presented within the framework of Non-Equilibrium Thermo Field Dynamics (NETFD). It is performed by introducing an appropriate martingale operator in the Schrödinger and the Heisenberg representations with fermionic and bosonic Brownian motions. In order to decide the double tilde conjugation rule and the thermal state conditions for fermions, a generalization of the system consisting of a vector field and Faddeev-Popov ghosts to dissipative open situations is carried out within NETFD.
\end{abstract}

Key words: Non-Equilibrium Thermo Field Dynamics, stochastic differential equations, martingale operator, fermionic Brownian motion, bosonic Brownian motion

PACS: 05.30.-d, 02.50.Ey

\section{Introduction}

In this paper we study time-dependent behavior of non-equilibrium quantum systems involving stochastic forces which can be boson or fermion type and are called quantum Brownian motion. Present consideration is an extension of previous analysis reported comprehensively by one of the authors [1] and is given in terms of Non-Equilibrium Thermo Field Dynamics (NETFD) $[2,3,4]$.

* Corresponding author

Email address: arimitsu@cm.ph.tsukuba.ac.jp (T. Arimitsu).

1 Present address: Institute for Molecular Science, Myodaiji, Okazaki 444-8585, Japan 
NETFD is a unified formalism, which enables us to treat dissipative quantum systems by the method similar to usual quantum mechanics or quantum field theory, which accommodates the concept of the dual structure in the interpretation of nature, i.e. in terms of the operator algebra and the representation space. The representation space in NETFD is composed of a direct product of two Hilbert spaces: one is for non-tilde fields, and the other for tilde fields. Within the statistical operator (density operator) formalism there is entanglement between operators and statistical operator due to their noncommutativity. Introduction of two kinds of operators, without tilde and with tilde, made it possible to resolve the entanglement between relevant operators and the statistical operator.

We are deriving a unified system of quantum stochastic differential equations (QSDEs) under the influence of quantum Brownian motion, including the quantum stochastic Liouville equation and the quantum Langevin equation. The quantum Fokker-Planck equation is derived by taking the random average of the corresponding stochastic Liouville equation. The relation between the Langevin equation and the stochastic Liouville equation, as well as between the Heisenberg equation for operators of gross variables and the quantum FokkerPlanck equation obtained here, is similar to the one between the Heisenberg equation and the Schrödinger equation in quantum mechanics and field theory. Our extension of analysis [1] consists of essentially three items. Two of them include definition of fermionic Brownian motion and treatment of fermions in NETFD, i.e. the tilde conjugation rule and the thermal state conditions in the case of fermion systems. Third item is the simultaneous consideration of hermitian and non-hermitian interaction Hamiltonians.

To begin with, we first remind briefly some standard steps that people usually take in order to obtain the irreversible evolution of macroscopic systems starting from the microscopic level. At present, there are many viewpoints giving us tools how to describe $N$-body systems out of equilibrium. At the same time, one usually follows one of several basic approaches: (i) the behavior of the systems is expressed in terms of not the total ( $N$-particle) distribution function but $s$-particle ones (with $s$ being usually 1 and/or 2), (ii) the dynamics of the systems is characterized by the evolution of a "coarse grained" phase-space distribution function or statistical operator, and (iii) the evolution of the systems is described by the equations of motion for the dynamical gross variables.

The approach (i) is intimately related to the Bogoliubov method of a reduced description of many-particle systems [5], which is widely used for construction of kinetic equations based on the Liouville or the Liouville-von-Neumann equation. Bogoliubov's hypothesis that the time dependence of higher-particle distribution functions enter through the one-particle distribution provides a fundamental importance in various schemes of truncation of the BBGKY hi- 
erarchy.

In the approach (ii), the most frequently used tools are projection operators introduced by Nakajima [6] and Zwanzig $[7,8]$. The basic idea underlying the application of their techniques to complex systems is to regard the operation of tracing over the environment as a formal projection in the space of the total system. It became especially popular in quantum optics where the so-called quantum master equation for reduced statistical operator of a relevant system now bears their names and is called the Nakajima-Zwanzig equation [9].

The general framework, called sub-dynamics, at the Brussels school is also related to the approach (ii) but the underlying concept is different from the one by Nakajima and Zwanzig. The main point here is the notion of the increase of the number of correlations within a system in time. It has been expounded in detail by Prigogine and coauthors, see e.g. [10,11].

Regarding to the approach (iii), we should mention projection operator by Mori [12] and the one by Kawasaki and Gunton [13]. The former is used to derive linear equations of motion for gross variables out of non-linear equations. Originally, one of the intentions to introduce such an operator was to obtain expressions for physical (measurable) kinetic coefficients. The latter is an improved version of the time-dependent projection operator by Robertson $[14]$.

Zubarev introduced the concept of non-equilibrium ensemble as a generalization of Green's works on the statistical mechanics of linear dissipation processes $[15,16]$ and Kubo's theory of linear response of systems to mechanical [17] and thermodynamical [18] external perturbations. This generalization is known as the method of non-equilibrium statistical operator [19]. It is shown that this has a close relationship to the projection operator methods [20].

Above mentioned methods do not exhaust the entire list, but they may be the most generic ones. However, in this paper we do not follow them. In the case of presence of additional degree(s) of freedom, e.g. stochastic force(s), description may be given also in some optional way (in a sense that consideration does not start from the very microscopic level). The theory of Brownian motion is an example. The fundamental equation here is the Langevin equation and it is the stochastic differential equation for dynamical variables [21,22]. Random forces in Langevin equation are usually described by Gaussian white stochastic processes. Stochastic integral with respect to such processes is defined as a kind of a Riemann-Stieltjes one [23] where multiplication between the stochastic increment and integrand is commonly considered in the form of Itô [24] or Stratonovich [25] (for Itô and Stratonovich multiplications see Appendix A).

The Langevin equation can be used to calculate various time correlation functions. Now it is radically extended to solve numerous problems in different 
areas $[26,27,28,29,30]$. In particular, the theory of Brownian motion itself has been extended to situations where the "Brownian particle" is not a real particle anymore, but instead some collective properties of a macroscopic system. Corresponding equation in the phase space or the Liouville space of statistical operators can be considered as a sort of stochastic differential equation too. In order to investigate classical stochastic systems, the stochastic Liouville equation was introduced first by Anderson [31] and Kubo [32,33,34].

There were several attempts to extend the classical theory (both the Langevin and the stochastic Liouville equations) for quantum cases. Study of the Langevin equation for quantum systems has its origin in papers by Senitzky [35,36,37], Schwinger [38], Haken [39,40,41,42] and Lax [43], where they investigated a quantum mechanical damped harmonic oscillator in connection with laser systems. In particular, it was shown that the quantum noise, i.e. the spontaneous emission, can be treated in a way similar to the thermal fluctuations, and that the noise source has non-zero second moments proportional to a quantity which can be associated with a quantum analog of a diffusion coefficient. As it was noticed by Kubo [44] in his discussion with van Kampen, the random force must be an operator defined in its own Hilbert space, which does not happen in classical case since there is no consideration of space for the random force.

Mathematical study of the quantum stochastic processes was initiated by Davies [45,46], Hudson [47,48,49,50,51,52], Accardi [53,54], Parthasarathy [55,56,57] and their co-authors. Quantum mechanical analogs of Wiener processes [47] and quantum Itô formula for boson systems [48,49,50,51] were defined first by Hudson et al.. The classical Brownian motion is replaced here by the pair of one-parameter unitary group authomorphisms, namely by the annihilation and creation boson random force operators with time indices in the boson Fock space, named quantum Brownian motion. Fermion stochastic calculus were defined by Applebaum, Hudson and Parthasarathy [58,59,60,61,62,63]. In these papers, they developed the fermion analog of the corresponding boson theory $[49,50]$ in which the annihilation and creation processes are fermion field operators in the fermion Fock space. Within the frame of this formalism, the Itô-Clifford integral $[64,65,66,67]$ - fermion analog of the classical Brownian motion - is contained as a special case. It should be noted, however, that in both boson and fermion theories of quantum stochastic calculus mathematicians were debating unitary processes only. For readers' convenience, clue of mathematicians' theory of quantum Brownian motion and the extension with allowance for thermal degree of freedom are put into Appendix B.

Contrary to expectations, attempts to extend the classical stochastic Liouville equation for quantum case were not very successful so far. In present work we construct our consideration using the formalism of NETFD. It is an alternative way to the above mentioned general methods of non-equilibrium 
statistical mechanics in the sense that it provides us with a general structure of the canonical operator formalism for dissipative non-equilibrium quantum systems without starting from the microscopic description, and turns out to be especially successful in the inclusion of quantum stochastic forces. In particular, a unified canonical operator formalism of QSDEs for boson systems was constructed first within NETFD $[68,69,70,71,72,73,1]$ on the basis of the quantum stochastic Liouville equation.

The paper is organized as follows. First, in section 2, we remind a brief essence of the formalism of NETFD by giving its technical basics and some fundamentals. In section 3 we derive the semi-free time evolution generator for systems in non-stationary case. The semi-free generator is bi-linear and globally gauge invariant. The annihilation and creation operators are introduced by means of a time-dependent Bogoliubov transformation. We close the section by calculating the two-point function. The generating functional method, which gives us the relation between the method of NETFD and the one of the Schwinger closed-time path, is introduced in section 4. Interaction with external fields is considered in section 5. Here we study two cases: hermitian and non-hermitian interaction hat-Hamiltonians. To make possible their simultaneous consideration we introduce an auxiliary parameter $\lambda$ which plays the rôle of a switch between the cases. In section 6 , the general expression of the stochastic semifree time evolution generator is derived for a non-stationary Gaussian white quantum stochastic process by means of the interaction hat-Hamiltonian with arbitrary $\lambda$. Correlations of the random force operators are also derived generally. With the generator, quantum stochastic Liouville equations and quantum stochastic Langevin equations of both Itô and Stratonovich types of the system are investigated in a unified manner. We conclude the section by deriving the equation of motion for the expectation value of an arbitrary operator of the relevant system. In section 7 , we consider a semi-free system with a stationary process and check explicitly the irreversibility of such a process in terms of its Boltzmann entropy. In section 8 we investigate relation to the Monte Carlo wave-function method. Summary and open questions are put into section 9. Auxiliary material is put into Appendices.

\section{Basics of NETFD}

Information about the general method of NETFD can be found in many papers and we refer first of all to the original source $[2,3,4]$ and the review article [1]. To make our paper self-contained, we include some standard steps which are

necessary at least to fix the notations. The formalism of NETFD is constructed upon the following fundamental requirements.

An arbitrary operator $A$ in NETFD is accompanied by its tilde conjugated 
partner $\tilde{A}$, called tilde operator, according to the rule

$$
\begin{aligned}
\left(A_{1} A_{2}\right)^{\sim} & =\tilde{A}_{1} \tilde{A}_{2}, \\
\left(c_{1} A_{1}+c_{2} A_{2}\right)^{\sim} & =c_{1}^{*} \tilde{A}_{1}+c_{2}^{*} \tilde{A}_{2}, \\
(\tilde{A})^{\sim} & =A,
\end{aligned}
$$

where $c_{1}$ and $c_{2}$ are $c$-numbers. It should be noted that in the present paper the double tilde conjugation rule (2.3) is of the same form for both bosonic and fermionic operators and leaves them unchanged.

To indicate commutation or anti-commutation of two operators, say $A_{1}$ and $A_{2}$, we will use the notation $\left[A_{1}, A_{2}\right\}$ and call it (anti-)commutator, which should be understood as

$$
\left[A_{1}, A_{2}\right\}=\left[A_{1}, A_{2}\right]_{+}=\left\{A_{1}, A_{2}\right\}=A_{1} A_{2}+A_{2} A_{1}
$$

when both operators are fermionic, or

$$
\left[A_{1}, A_{2}\right\}=\left[A_{1}, A_{2}\right]_{-}=\left[A_{1}, A_{2}\right]=A_{1} A_{2}-A_{2} A_{1}
$$

otherwise. $^{2}$

Tilde and non-tilde operators, say $A_{1}$ and $\tilde{A}_{2}$, are supposed to be mutually (anti-)commutative at equal time, i.e.

$$
\left[A_{1}, \tilde{A}_{2}\right\}=0
$$

Tilde and non-tilde operators are related with each other through the thermal state condition (TSC)

$$
\langle\theta| \tilde{A}^{\dagger}=\tau^{*}\langle\theta| A
$$

where $\langle\theta|$ represents the thermal bra-vacuum; $\tau$ is the complex parameter which takes two values:

$$
\tau= \begin{cases}1 & \text { for bosonic operators } \\ i & \text { for fermionic operators }\end{cases}
$$

$\overline{2}$ When one operator is bosonic and another one is fermionic, the rule of commutation depends on the system. In this paper for such combinations we assume $(2.5)$. 
Derivation of the double tilde conjugation rule and TSC for fermionic operators used in this paper is given in Appendix C.

Within the framework of NETFD, the dynamical evolution of a system is described by the Schrödinger equation (here we use the system with $\hbar=1$ )

$$
\frac{\partial}{\partial t}|0(t)\rangle=-i \hat{H}|0(t)\rangle
$$

where $|O(t)\rangle$ represents the thermal ket-vacuum. It can be also called the quantum master equation or the quantum Fokker-Planck equation in this paper. The thermal vacuums are tilde invariant, i.e., $\left\langle\left.\theta\right|^{\sim}=\langle\theta| \text { and } \mid 0(t)\right\rangle^{\sim}=|0(t)\rangle$, and are normalized as $\langle\theta \mid O(t)\rangle=1$. The hat-Hamiltonian $\hat{H}$, an infinitesimal time-evolution generator, satisfies the tildian condition:

$$
(i \hat{H})^{\sim}=i \hat{H}
$$

The tildian hat-Hamiltonian is not necessarily hermitian operator. It has zero eigenvalues for the thermal bra-vacuum

$$
\langle\theta| \hat{H}=0,
$$

which is nothing but manifestations of conservation of probability.

Introducing the time-evolution operator $\hat{V}(t)$ by

$$
\frac{d}{d t} \hat{V}(t)=-i \hat{H} \hat{V}(t)
$$

with the initial condition $\hat{V}(0)=1$, we can define the Heisenberg operator

$$
A(t)=\hat{V}^{-1}(t) A \hat{V}(t)
$$

satisfying the Heisenberg equation for dissipative systems

$$
\frac{d}{d t} A(t)=i[\hat{H}(t), A(t)]
$$

where $\hat{H}(t)$ is the hat-Hamiltonian in the Heisenberg representation. The existence of the Heisenberg equation of motion for coarse grained operators enables us to construct a canonical formalism of the dissipative quantum field theory. Note that with the help of TSC we have an equation of motion for a vector $\langle\theta| A(t)$ 


$$
\frac{d}{d t}\langle\theta| A(t)=i\langle\theta|[\hat{H}(t), A(t)]
$$

in terms of only non-tilde operators. The expectation value of an observable operator $A$ at time $t$ is given by

$$
\langle A(t)\rangle=\langle\theta|A| 0(t)\rangle=\langle\theta|A(t)| 0\rangle,
$$

where $|0\rangle=|0(t=0)\rangle$. We define that observable operators consist only of non-tilde operators. ${ }^{3}$

\section{Semi-free hat-Hamiltonian}

Let us consider a system specified by the total hat-Hamiltonian

$$
\hat{H}_{t}^{\text {tot }}=\hat{H}_{t}+\hat{H}_{1}+\hat{H}_{\mathrm{I}, t}
$$

where $\hat{H}_{t}$ is a semi-free hat-Hamiltonian, whereas $\hat{H}_{1}$ and $\hat{H}_{\mathrm{I}, t}$ are, respectively, the interaction hat-Hamiltonian within the relevant system and the one representing the coupling with external fields. The system itself is supposed to be consistent with all the requirements of NETFD given in the previous section. Some general remark about treatment of interaction within the relevant system is given in section 4. Explicit treatment of interaction with external fields is given in section 5. Here we concentrate on derivation and study of properties of the semi-free hat-Hamiltonian, i.e. renormalized unperturbed hat-Hamiltonian.

\subsection{Derivation of the semi-free hat-Hamiltonian}

The semi-free hat-Hamiltonian is bilinear in operators $a, a^{\dagger}, \tilde{a}$ and $\tilde{a}^{\dagger}$, and is invariant under the phase transformation $a \rightarrow a \mathrm{e}^{i \phi}$ :

$$
\hat{H}_{t}=h_{1}(t) a^{\dagger} a+h_{2}(t) \tilde{a}^{\dagger} \tilde{a}+h_{3}(t) a \tilde{a}+h_{4}(t) a^{\dagger} \tilde{a}^{\dagger}+h_{0}(t)
$$

where $h_{j}(t)$ are time-dependent complex $c$-number functions. Operators $a, a^{\dagger}$, $\tilde{a}$ and $\tilde{a}^{\dagger}$ satisfy the canonical (anti-)commutation relations

3 We can include tilde operators in addition to non-tilde ones in the definition of observable. However, inclusion of tilde operators may give us a set of different but equivalent definitions for one observable operator. 


$$
\left[a_{\mathbf{k}}, a_{\mathbf{k}^{\prime}}^{\dagger}\right]_{-\sigma}=\delta_{\mathbf{k}, \mathbf{k}^{\prime}}, \quad\left[\tilde{a}_{\mathbf{k}}, \tilde{a}_{\mathbf{k}^{\prime}}^{\dagger}\right]_{-\sigma}=\delta_{\mathbf{k}, \mathbf{k}^{\prime}},
$$

where we use $\sigma=1$ for bosonic systems and $\sigma=-1$ for fermionic ones. According to (2.6), tilde and non-tilde operators are mutually (anti-)commutative. In the following account, a subscript $\mathbf{k}$ for specifying a momentum and/or other degrees of freedom will be dropped unless it is necessary. Number of unknown functions $h_{j}(t)$ can be reduced by the use of (2.11) and tildian (2.10) for the semi-free hat-Hamiltonian. It results in

$$
\hat{H}_{t}=\hat{H}_{\mathrm{S}, t}+i \hat{\Pi}_{t},
$$

where

$$
\begin{aligned}
\hat{H}_{\mathrm{S}, t}= & \omega(t)\left(a^{\dagger} a-\tilde{a}^{\dagger} \tilde{a}\right), \\
\hat{\Pi}_{t}= & c_{1}(t)\left(a^{\dagger} a+\tilde{a}^{\dagger} \tilde{a}\right)-\tau\left[2 c_{1}(t)+c_{2}(t)\right] a^{\dagger} \tilde{a}^{\dagger} \\
& +\sigma \tau c_{2}(t) a \tilde{a}+\sigma\left[2 c_{1}(t)+c_{2}(t)\right],
\end{aligned}
$$

with

$$
\begin{aligned}
\omega(t) & =\Re \mathfrak{e} h_{1}(t), \\
c_{1}(t) & =\Im \mathfrak{m} h_{1}(t), \\
c_{2}(t) & =\left\{\begin{array}{l}
\Im \mathfrak{m} h_{3}(t) \text { for bosonic systems, } \\
\Re \mathfrak{e} h_{3}(t) \text { for fermionic systems. }
\end{array}\right.
\end{aligned}
$$

Let us introduce operators $a(t)$ and $a^{\dagger}(t)$ in the interaction representation defined by

$$
a(t)=\hat{V}^{-1}(t) a \hat{V}(t), \quad a^{\dagger}(t)=\hat{V}^{-1}(t) a^{\dagger} \hat{V}(t),
$$

where

$$
\frac{d}{d t} \hat{V}(t)=-i \hat{H}_{t} \hat{V}(t)
$$

with the initial condition $\hat{V}(0)=1$. They satisfy the equal-time (anti-)commutation relations

$$
\left[a(t), a^{\dagger}(t)\right]_{-\sigma}=1 .
$$


The Heisenberg equation (2.14) for $a(t)$ and $a^{\dagger}(t)$ with

$$
\hat{H}(t)=\hat{V}^{-1}(t) \hat{H}_{t} \hat{V}(t)
$$

are explicitly given by

$$
\begin{aligned}
\frac{d a(t)}{d t} & =\left[c_{1}(t)-i \omega(t)\right] a(t)-\tau\left[2 c_{1}(t)+c_{2}(t)\right] \tilde{a}^{\dagger}(t), \\
\frac{d a^{\dagger}(t)}{d t} & =\left[i \omega(t)-c_{1}(t)\right] a^{\#}(t)-\tau c_{2}(t) \tilde{a}(t) .
\end{aligned}
$$

In these formulae we used a symbol tt instead of usual dagger because the semi-free hat-Hamiltonian $\hat{H}_{t}$ is not necessarily hermitian.

Since the semi-free hat-Hamiltonian $\hat{H}_{t}$ satisfies $(2.11)$, we have TSC for the bra-vacuum at time $t$

$$
\langle\theta| \tilde{a}^{\dagger}(t)=\tau^{*}\langle\theta| a(t)
$$

By making use of the Heisenberg equations (3.14) and (3.15), and of TSC (3.16), one obtains the equation of motion for a vector $\langle\theta| a^{\dagger}(t) a(t)$ in the form

$$
\frac{d}{d t}\langle\theta| a^{\#}(t) a(t)=-2 \kappa(t)\langle\theta| a^{\#}(t) a(t)+i \Sigma^{<}(t)\langle\theta|,
$$

where $\kappa(t)$ and $i \Sigma^{<}(t)$ are defined by

$$
\begin{aligned}
\kappa(t) & =c_{1}(t)+c_{2}(t), \\
i \Sigma^{<}(t) & =-\sigma\left[2 c_{1}(t)+c_{2}(t)\right] .
\end{aligned}
$$

Substituting (3.18) and (3.19) into (3.14) and (3.15) one gets equations of motion for operators $a(t)$ and $a^{\#}(t)$ in the form

$$
\begin{aligned}
\frac{d a(t)}{d t} & =-[i \omega(t)+\kappa(t)] a(t)-\sigma i \Sigma^{<}(t)\left[a(t)-\tau \tilde{a}^{\dagger}(t)\right] \\
\frac{d a^{\#}(t)}{d t} & =[i \omega(t)+\kappa(t)] a^{\#}(t)+\sigma i \Sigma^{<}(t)\left[a^{\#}(t)-\tau \tilde{a}(t)\right]-2 \tau \kappa(t) \tilde{a}(t)
\end{aligned}
$$

Applying the thermal ket-vacuum $|0\rangle$ at the initial time to (3.17), we obtain the equation of motion for the one-particle distribution function 


$$
n(t)=\left\langle\theta\left|a^{\#}(t) a(t)\right| 0\right\rangle
$$

in the form

$$
\frac{d}{d t} n(t)=-2 \kappa(t) n(t)+i \Sigma^{<}(t) .
$$

Equation (3.23) can be identified as the generalized Boltzmann equation of the system. The function $i \Sigma^{<}(t)$ is given when the interaction hat-Hamiltonian $\hat{H}_{1}$ is defined.

The initial ket-vacuum $|0\rangle$ is specified by TSC

$$
\tilde{a}|0\rangle=\tau f a^{\dagger}|0\rangle
$$

with $f \in \mathbf{R}$. The initial value for the one-particle distribution function $n=$ $n(t=0)$ is determined by $f$. Since

$$
\begin{aligned}
n & =n^{*}=\left\langle\theta\left|a^{\dagger} a\right| 0\right\rangle^{\sim}=\left\langle\theta\left|\tilde{a}^{\dagger} \tilde{a}\right| 0\right\rangle=\tau^{*}\langle\theta|a \tilde{a}| 0\rangle=|\tau|^{2} f\left\langle\theta\left|a a^{\dagger}\right| 0\right\rangle \\
& =f[1+\sigma n],
\end{aligned}
$$

we have

$$
n=f[1-\sigma f]^{-1} .
$$

In the first equality of (3.25) we used the fact that $n$ is a real number; in the third equality we used the tilde invariance of the thermal vacuums $\langle\theta|$ and $|0\rangle$; finally, in the fourth and fifth equalities we used TSCs (2.7) and (3.24), respectively.

Solving the Heisenberg equations for $a(t), a^{\#}(t)$ and their tilde conjugates, and using TSC at initial time (3.24), we find TSC for the ket-vacuum at time $t$

$$
\tilde{a}(t)|0\rangle=\frac{\tau n(t)}{1+\sigma n(t)} a^{\dagger}(t)|0\rangle,
$$

where $n(t)$ satisfies the Boltzmann equation (3.23).

Substituting (3.18), (3.19) and (3.23) into (3.6) one gets the most general form of $\hat{\Pi}_{t}$ in the interaction representation:

$$
\hat{\Pi}_{t}=-\{\kappa(t)[1+2 \sigma n(t)]+\sigma \dot{n}(t)\}\left(a^{\dagger} a+\tilde{a}^{\dagger} \tilde{a}\right)
$$




$$
\begin{aligned}
& +\sigma \tau\{2 \kappa(t)[1+\sigma n(t)]+\sigma \dot{n}(t)\} a \tilde{a} \\
& +\sigma \tau\{2 \kappa(t) n(t)+\dot{n}(t)\} a^{\dagger} \tilde{a}^{\dagger}-\{2 \kappa(t) n(t)+\dot{n}(t)\}
\end{aligned}
$$

Here, we used the abbreviation $\dot{n}(t)=d n(t) / d t$.

By introducing thermal doublet notations

$$
\bar{a}^{\nu}=\left(a^{\dagger},-\tau \tilde{a}\right), \quad a^{\mu}=\text { collon }\left(a, \tau \tilde{a}^{\dagger}\right)
$$

canonical (anti-)commutation relations are written as

$$
\left[a^{\mu}, \bar{a}^{\nu}\right]_{-\sigma}=\delta^{\mu \nu}
$$

The resulting semi-free hat-Hamiltonian (3.4) can be presented in a compact form as

$$
\hat{H}_{t}=\omega(t) \bar{a}^{\mu} a^{\mu}+i \bar{a}^{\mu} A(t)^{\mu \nu} a^{\nu}+\sigma[\omega(t)+i \kappa(t)]
$$

where matrix $A(t)^{\mu \nu}$ has the following structure:

$$
A(t)^{\mu \nu}=\sigma\left(\begin{array}{lr}
-\kappa(t)[2 n(t)+\sigma]-\dot{n}(t), & 2 \kappa(t) n(t)+\dot{n}(t) \\
-2 \kappa(t)[n(t)+\sigma]-\dot{n}(t), \kappa(t)[2 n(t)+\sigma]+\dot{n}(t)
\end{array}\right)
$$

\subsection{Annihilation and creation operators}

Let us introduce annihilation and creation operators by

$$
\begin{aligned}
\gamma(t) & =[1+\sigma n(t)] a(t)-\sigma \tau n(t) \tilde{a}^{\dagger}(t), \\
\tilde{\gamma}^{+}(t) & =\tilde{a}^{\dagger}(t)-\sigma \tau a(t) .
\end{aligned}
$$

From TSCs (3.16) and (3.27) at time $t$, we see that they annihilate the vacuums:

$$
\begin{array}{ll}
\langle\theta| \gamma^{\text {क }}(t)=0, & \gamma(t)|0\rangle=0, \\
\langle\theta| \tilde{\gamma}^{\text {f }}(t)=0, & \tilde{\gamma}(t)|0\rangle=0 .
\end{array}
$$

With the thermal doublet notations 


$$
\begin{array}{ll}
\bar{a}(t)^{\mu}=\left(a^{\dagger}(t),-\tau \tilde{a}(t)\right), & a(t)^{\nu}=\text { collon }\left(a(t), \tau \tilde{a}^{\dagger}(t)\right), \\
\bar{\gamma}(t)^{\nu}=\left(\gamma^{\dagger}(t),-\tau \tilde{\gamma}(t)\right), & \gamma(t)^{\mu}=\text { collon }\left(\gamma(t), \tau \tilde{\gamma}^{\dagger}(t)\right),
\end{array}
$$

(3.33), (3.34) and their tilde conjugates can be written as

$$
\bar{\gamma}(t)^{\nu}=\bar{a}(t)^{\mu}\left[B^{-1}(t)\right]^{\mu \nu}, \quad \gamma(t)^{\mu}=B(t)^{\mu \nu} a(t)^{\nu},
$$

where $B(t)^{\mu \nu}$ is a matrix of the time-dependent Bogoliubov transformation:

$$
B(t)^{\mu \nu}=\left(\begin{array}{cc}
1+\sigma n(t) & -\sigma n(t) \\
-1 & 1
\end{array}\right)
$$

This transformation is the canonical one since it leaves the canonical (anti-)commutation relations unchanged:

$$
\left[\gamma(t)^{\mu}, \bar{\gamma}(t)^{\nu}\right]_{-\sigma}=\delta^{\mu \nu}
$$

The equation of motion for the thermal doublet $\gamma(t)^{\mu}$ is derived as

$$
\frac{d}{d t} \gamma(t)^{\mu}=\left[-i \omega(t) \delta^{\mu \nu}-\kappa(t) \tau_{3}^{\mu \nu}\right] \gamma(t)^{\nu}
$$

where

$$
\tau_{3}^{\mu \nu}=\left(\begin{array}{rr}
1 & 0 \\
0 & -1
\end{array}\right)
$$

The solution of (3.42) then is obtained in the form

$$
\gamma(t)^{\mu}=\exp \left\{\int_{0}^{t} d t^{\prime}\left[-i \omega\left(t^{\prime}\right) \delta^{\mu \nu}-\kappa\left(t^{\prime}\right) \tau_{3}^{\mu \nu}\right]\right\} \gamma(0)^{\nu}
$$

\subsection{Schrödinger representation}

Annihilation and creation operators in the Schrödinger representation are introduced by the relations 


$$
\bar{\gamma}(t)^{\nu}=\hat{V}^{-1}(t) \bar{\gamma}_{t}^{\nu} \hat{V}(t), \quad \gamma(t)^{\mu}=\hat{V}^{-1}(t) \gamma_{t}^{\mu} \hat{V}(t),
$$

with $\hat{V}(t)$ being specified by (3.11) and the thermal doublet notations

$$
\bar{\gamma}_{t}^{\nu}=\left(\gamma^{+},-\tau \tilde{\gamma}_{t}\right), \quad \gamma_{t}^{\mu}=\text { collon }\left(\gamma_{t}, \tau \tilde{\gamma}^{\text {q }}\right)
$$

Using (3.29), one can write

$$
\bar{\gamma}_{t}^{\nu}=\bar{a}^{\mu}\left[B^{-1}(t)\right]^{\mu \nu}, \quad \gamma_{t}^{\mu}=B(t)^{\mu \nu} a^{\nu}
$$

where matrix $B(t)^{\mu \nu}$ is given by (3.40).

We see that the annihilation and creation operators in the Schrödinger representation annihilate the vacuums at time $t$ :

$$
\begin{array}{ll}
\langle\theta| \gamma^{+}=0, & \gamma_{t}|O(t)\rangle=0, \\
\langle\theta| \tilde{\gamma}^{\text {+ }}=0, & \tilde{\gamma}_{t}|O(t)\rangle=0 .
\end{array}
$$

Note that creation operator $\gamma^{q}$ and its tilde conjugated partner $\tilde{\gamma}^{+}$do not depend on time. It is consistent with the fact that the vacuum $\langle\theta|$ does not depend on time due to the property $\langle\theta| \hat{H}_{t}=0$.

The terms (3.5) and (3.28) of the semi-free hat-Hamiltonian $\hat{H}_{t},(3.4)$, now read

$$
\begin{aligned}
\hat{H}_{\mathrm{S}, t} & =\omega(t)\left(\gamma^{+} \gamma_{t}-\tilde{\gamma}^{\text {q}} \tilde{\gamma}_{t}\right), \\
\hat{\Pi}_{t} & =-\kappa(t)\left(\gamma^{+} \gamma_{t}+\tilde{\gamma}^{\text {q }} \tilde{\gamma}_{t}\right)+\sigma \tau \dot{n}(t) \gamma^{\text {q }} \tilde{\gamma}^{\text {q }},
\end{aligned}
$$

in the normal ordering with respect to the annihilation and creation operators in the Schrödinger representation. When the system is semi-free, putting (3.50) and (3.51) into (3.4) and substituting $\hat{H}_{t}$ for $\hat{H}$ into the Schrödinger equation (2.9), one has

$$
\frac{\partial}{\partial t}|O(t)\rangle=\sigma \tau \dot{n}(t) \gamma^{\text {क }} \tilde{\gamma}^{\text {+ }}|0(t)\rangle
$$

It is solved to give

$$
|0(t)\rangle=\exp \left\{-\left\langle\theta\left|\tilde{\gamma}_{t} \gamma_{t}\right| 0\right\rangle \gamma^{\text {क }} \tilde{\gamma}^{\text {क }}\right\}|0\rangle .
$$

Here we introduced a kind of the order parameter 


$$
\left\langle\theta\left|\tilde{\gamma}_{t} \gamma_{t}\right| 0\right\rangle=\sigma \tau[n(0)-n(t)]
$$

which gives a measure of difference of the system from the initial state. From (3.53) we see that the evolution of the ket-vacuum is realized by a condensation of tilde and non-tilde particle pairs into initial ket-vacuum. The ket-vacuum itself is the functional of the one-particle distribution function $n(t)$. The dependence of the thermal ket-vacuum on $n(t)$ is given by

$$
\frac{\delta}{\delta n(t)}|0(t)\rangle=\sigma \tau \gamma^{\text {q }} \tilde{\gamma}^{\text {q }}|0(t)\rangle .
$$

Then the Schrödinger equation can be written in an alternative way:

$$
\left\{\frac{\partial}{\partial t}-\dot{n}(t) \frac{\delta}{\delta n(t)}\right\}|0(t)\rangle=0 \text {. }
$$

This shows that the vacuum $|O(t)\rangle$ is migrating in the super-representation space spanned by the one-particle distribution function $\left\{n_{\mathbf{k}}(t)\right\}$ with the velocity $\left\{\dot{n}_{\mathbf{k}}(t)\right\}$ as a conserved quantity $[74,75]$.

\subsection{Two-point function of the semi-free field}

A time-ordered two-point function $G\left(t, t^{\prime}\right)^{\mu \nu}$ (propagator), defined by

$$
G\left(t, t^{\prime}\right)^{\mu \nu}=-i\left\langle\theta\left|T\left[a(t)^{\mu} \bar{a}\left(t^{\prime}\right)^{\nu}\right]\right| 0\right\rangle,
$$

is given by

$$
G\left(t, t^{\prime}\right)^{\mu \nu}=\left[B^{-1}(t)\right]^{\mu \lambda} \mathcal{G}\left(t, t^{\prime}\right)^{\lambda \rho} B\left(t^{\prime}\right)^{\rho \nu},
$$

with

$$
\mathcal{G}\left(t, t^{\prime}\right)^{\lambda \rho}=-i\left\langle\theta\left|T\left[\gamma(t)^{\lambda} \bar{\gamma}\left(t^{\prime}\right)^{\rho}\right]\right| 0\right\rangle=\left(\begin{array}{ll}
G^{\mathrm{R}}\left(t, t^{\prime}\right) & 0 \\
0 & G^{\mathrm{A}}\left(t, t^{\prime}\right)
\end{array}\right)
$$

where non-zero matrix elements are

$$
\begin{aligned}
G^{\mathrm{R}}\left(t, t^{\prime}\right) & =-i\left\langle\theta\left|T\left[\gamma(t) \gamma^{+}\left(t^{\prime}\right)\right]\right| 0\right\rangle \\
& =-i \theta\left(t-t^{\prime}\right) \exp \left\{\int_{t^{\prime}}^{t} d t^{\prime \prime}\left[-i \omega\left(t^{\prime \prime}\right)-\kappa\left(t^{\prime \prime}\right)\right]\right\},
\end{aligned}
$$




$$
\begin{aligned}
G^{\mathrm{A}}\left(t, t^{\prime}\right) & =i \sigma\left\langle\theta\left|T\left[\tilde{\gamma}^{+}(t) \tilde{\gamma}\left(t^{\prime}\right)\right]\right| 0\right\rangle \\
& =i \theta\left(t^{\prime}-t\right) \exp \left\{\int_{t}^{t^{\prime}} d t^{\prime \prime}\left[i \omega\left(t^{\prime \prime}\right)-\kappa\left(t^{\prime \prime}\right)\right]\right\} .
\end{aligned}
$$

Here, $T$ is the time ordering operator.

\section{Generating functional method}

Let us define the generating functional for the semi-free field by

$$
Z[K, \tilde{K}]=\left\langle\theta\left|T \exp \left\{-i \int_{0}^{\bar{t}} d t S(t)\right\}\right| 0\right\rangle,
$$

where the source function $S(t)$ reads

$$
S(t)=\bar{K}(t)^{\mu} a(t)^{\mu}+\bar{a}(t)^{\mu} K(t)^{\mu}=\bar{K}_{\gamma}(t)^{\mu} \gamma(t)^{\mu}+\bar{\gamma}(t)^{\mu} K_{\gamma}(t)^{\mu},
$$

with

$$
\bar{K}(t)^{\mu}=\left(K(t)^{*},-\tau \tilde{K}(t)\right), \quad K(t)^{\nu}=\operatorname{collon}\left(K(t), \tau \tilde{K}(t)^{*}\right),
$$

and similar notations for $\bar{K}_{\gamma}(t)^{\nu}$ and $K_{\gamma}(t)^{\mu}$. The $K$ 's are related by the Bogoliubov transformation

$$
\bar{K}_{\gamma}(t)^{\nu}=\bar{K}(t)^{\mu}\left[B^{-1}(t)\right]^{\mu \nu}, \quad K_{\gamma}(t)^{\mu}=B(t)^{\mu \nu} K(t)^{\nu} .
$$

Matrix $B(t)^{\mu \nu}$ here is the one given by (3.40). External fictitious fields $K(t)^{\mu}$, $\bar{K}(t)^{\nu}$ are $c$-numbers or Grassmann numbers corresponding to $\sigma=1$ or $\sigma=$ -1 , and satisfy

$$
\left[K(t)^{\mu}, \bar{K}(t)^{\nu}\right]_{-\sigma}=0 .
$$

Operators $a(t)^{\mu}, \bar{a}(t)^{\nu}, \gamma(t)^{\mu}$ and $\bar{\gamma}(t)^{\nu}$ are those in the interaction representation introduced in section 3 .

Taking the functional derivative of the generating functional (4.1), one has

$$
\delta \ln Z[K, \tilde{K}]=-i \int_{0}^{\bar{t}} d t\left[\delta \bar{K}_{\gamma}(t)^{\mu}\left\langle\gamma(t)^{\mu}\right\rangle_{K}+\left\langle\bar{\gamma}(t)^{\mu}\right\rangle_{K} \delta K_{\gamma}(t)^{\mu}\right]
$$


where $\left\langle\gamma(t)^{\mu}\right\rangle_{K}$ and $\left\langle\bar{\gamma}(t)^{\mu}\right\rangle_{K}$ are defined by

$$
\begin{aligned}
\left\langle\gamma(t)^{\mu}\right\rangle_{K} & =i \frac{\delta}{\delta \bar{K}_{\gamma}(t)^{\mu}} \ln Z[K, \tilde{K}] \\
& =\frac{1}{Z[K, \tilde{K}]}\left\langle\theta\left|T\left[\gamma(t)^{\mu} \exp \left\{-i \int_{0}^{\bar{t}} d t^{\prime} S\left(t^{\prime}\right)\right\}\right]\right| 0\right\rangle \\
\left\langle\bar{\gamma}(t)^{\mu}\right\rangle_{K} & =\sigma i \frac{\delta}{\delta K_{\gamma}(t)^{\mu}} \ln Z[K, \tilde{K}] \\
& =\frac{1}{Z[K, \tilde{K}]}\left\langle\theta\left|T\left[\bar{\gamma}(t)^{\mu} \exp \left\{-i \int_{0}^{\bar{t}} d t^{\prime} S\left(t^{\prime}\right)\right\}\right]\right| 0\right\rangle .
\end{aligned}
$$

The equation of motion for $\left\langle\gamma(t)^{\mu}\right\rangle_{K}$ is obtained in the form

$$
\frac{d}{d t}\left\langle\gamma(t)^{\mu}\right\rangle_{K}=\left[-i \omega(t) \delta^{\mu \nu}-\kappa(t) \tau_{3}^{\mu \nu}\right]\left\langle\gamma(t)^{\nu}\right\rangle_{K}-i K_{\gamma}(t)^{\mu}
$$

With the boundary conditions

$$
\begin{aligned}
\left\langle\gamma(0)^{\mu=1}\right\rangle_{K} & =\langle\gamma(0)\rangle_{K}=0, \\
\left\langle\gamma(\bar{t})^{\mu=2}\right\rangle_{K} & =\tau\left\langle\tilde{\gamma}^{+}(\bar{t})\right\rangle_{K}=0, \\
\left\langle\bar{\gamma}(\bar{t})^{\mu=1}\right\rangle_{K} & =\left\langle\gamma^{+}(\bar{t})\right\rangle_{K}=0, \\
\left\langle\bar{\gamma}(0)^{\mu=2}\right\rangle_{K} & =-\tau\langle\tilde{\gamma}(0)\rangle_{K}=0,
\end{aligned}
$$

it can be solved as

$$
\left\langle\gamma(t)^{\mu}\right\rangle_{K}=\int_{0}^{\bar{t}} d t^{\prime} \mathcal{G}\left(t, t^{\prime}\right)^{\mu \nu} K_{\gamma}\left(t^{\prime}\right)^{\nu}
$$

where $\mathcal{G}\left(t, t^{\prime}\right)^{\mu \nu}$ is given by (3.59). The boundary conditions in (4.10) to (4.13) are derived by TSCs (3.35) and (3.36).

Substituting (4.14) into (4.6), one finally obtains [76]

$$
\begin{aligned}
Z[K, \tilde{K}] & =\exp \left\{-i \int_{0}^{\bar{t}} d t \int_{0}^{\bar{t}} d t^{\prime} \bar{K}_{\gamma}(t)^{\mu} \mathcal{G}\left(t, t^{\prime}\right)^{\mu \nu} K_{\gamma}\left(t^{\prime}\right)^{\nu}\right\} \\
& =\exp \left\{-i \int_{0}^{\bar{t}} d t \int_{0}^{\bar{t}} d t^{\prime} \bar{K}(t)^{\mu} G\left(t, t^{\prime}\right)^{\mu \nu} K\left(t^{\prime}\right)^{\nu}\right\} .
\end{aligned}
$$


This expression has been derived first by Schwinger for a boson system within the closed-time path method [38]. Derivation of this result shown in the present section reveals the relation between the quantum operator formalism of dissipative fields (realized for the first time within NETFD) and their path integral formalism [38].

The effect of the interaction $\hat{H}_{1} \equiv \hat{H}_{1}\left(a^{\mu}, \bar{a}^{\nu}\right)$ within the system, which induces the dynamical correlations, can be taken into account by the generating functional

$$
Z_{1}[K, \tilde{K}]=\exp \left\{-i \int_{0}^{\bar{t}} d t \hat{H}_{1}\left(i \frac{\delta}{\delta \bar{K}(t)^{\mu}}, \sigma i \frac{\delta}{\delta K(t)^{\nu}}\right)\right\} Z[K, \tilde{K}]
$$

Note that $\hat{H}_{1}$ should satisfy $\langle\theta| \hat{H}_{1}=0$.

\section{Interaction with external fields}

\subsection{Hermitian interaction hat-Hamiltonian}

The simplest hat-Hamiltonian representing an interaction with an external field may be given by

$$
\hat{H}_{t}^{\prime}=H_{t}^{\prime}-\tilde{H}_{t}^{\prime}
$$

with a hermitian interaction Hamiltonian

$$
H_{t}^{\prime}=i\left(a^{\dagger} b_{t}-b_{t}^{\dagger} a\right)
$$

where $b_{t}, b_{t}^{\dagger}$ and their tilde conjugates are operators of the external system and are assumed to be (anti-)commutative with operators $a, a^{\dagger}$ and their tilde conjugates of the relevant system. The subscript $t$ indicates that these operators may depend on time. Note that the hat-Hamiltonian (5.1) is tildian, i.e.

$$
\left(i \hat{H}_{t}^{\prime}\right)^{\sim}=i \hat{H}_{t}^{\prime}
$$

The tilde and non-tilde operators of the external system are related with each other by 


$$
\langle| \tilde{b}_{t}^{\dagger}=\tau^{*}\langle| b_{t}
$$

where $\langle$ is the bra-vacuum for the external system. Applying the bra-vacuum $\langle\theta|$ for the relevant system on (5.1), one has

$$
\langle\theta| \hat{H}_{t}^{\prime}=-i\langle\theta|\left(\beta_{t}^{\text {क }} a+\sigma \tau \tilde{\beta}_{t}^{\text {q }} a^{\dagger}\right) .
$$

Here we introduced a new operator

$$
\beta_{t}^{\text {क }}=b_{t}^{\dagger}-\tau \tilde{b}_{t}
$$

which annihilates the bra-vacuum \langle| for the external system:

$$
\langle| \beta_{t}^{\text {q }}=0
$$

As it is seen from (5.6), the subscript $t$ of the new operator $\beta_{t}^{\text {q }}$ has been inherited from the original operators of the external system. By applying the bra-vacuum \langle| on $\hat{H}_{t}^{\prime}$ in addition to $\langle\theta|$, we observe that

$$
\left\langle\langle\theta| \hat{H}_{t}^{\prime}=0\right.
$$

where the bra-vacuum of a total system is introduced by

$$
\langle\langle\theta|=\langle| \cdot\langle\theta| .
$$

The dynamics of the system is described by the Schrödinger equation for the ket-vacuum $|O(t)\rangle\rangle$ of the whole system:

$$
\left.\left.\frac{\partial}{\partial t}|O(t)\rangle\right\rangle=-i \hat{H}_{t}^{\mathrm{tot}}|O(t)\rangle\right\rangle
$$

where $\hat{H}_{\mathrm{I}, t}$ in $\hat{H}_{t}^{\text {tot }}$ is replaced by $\hat{H}_{t}^{\prime}$. Conservation of the probability is guaranteed by $\left\langle\langle\theta| \hat{H}_{t}^{\text {tot }}=0\right.$ for the total system, i.e. the relevant system and the external system.

\subsection{Non-Hermitian interaction hat-Hamiltonian}

Let us consider if we can have an interaction hat-Hamiltonian which satisfies the conservation of probability within the relevant system. This feature is 
consistent with the one we have in the case of stochastic differential equations for classical systems.

We assume that the interaction hat-Hamiltonian is globally gauge invariant and bilinear:

$$
\begin{aligned}
\hat{H}_{t}^{\prime \prime}= & i\left\{h_{1} a^{\dagger} b_{t}+h_{2} a^{\dagger} \tilde{b}_{t}^{\dagger}+h_{3} \tilde{a} b_{t}+h_{4} \tilde{a} \tilde{b}_{t}^{\dagger}\right. \\
& \left.+h_{5} \tilde{a}^{\dagger} \tilde{b}_{t}+h_{6} \tilde{a}^{\dagger} b_{t}^{\dagger}+h_{7} a \tilde{b}_{t}+h_{8} a b_{t}^{\dagger}\right\},
\end{aligned}
$$

where quantities $h_{j}(j=1, \cdots, 8)$ are time-independent complex $c$-numbers. The tildian

$$
\left(i \hat{H}_{t}^{\prime \prime}\right)^{\sim}=i \hat{H}_{t}^{\prime \prime}
$$

gives us

$$
h_{1}^{*}=h_{5}, \quad h_{2}^{*}=h_{6}, \quad h_{3}^{*}=h_{7}, \quad h_{4}^{*}=h_{8} .
$$

By applying $\langle\theta|$ from the left to the Schrödinger equation

$$
\left.\left.\frac{\partial}{\partial t}|O(t)\rangle\right\rangle=-i \hat{H}_{t}^{\mathrm{tot}}|O(t)\rangle\right\rangle
$$

with $\hat{H}_{\mathrm{I}, t}$ in $\hat{H}_{t}^{\text {tot }}$ being replaced by $\hat{H}_{t}^{\prime \prime}$, we see that the requirement of the conservation of probability within the relevant system leads to

$$
\langle\theta| \hat{H}_{t}^{\prime \prime}=0
$$

$\hat{H}_{t}$ in $\hat{H}_{t}^{\text {tot }}$ is the semi-free hat-Hamiltonian of the relevant system satisfying (2.11). From (5.15) we obtain

$$
\begin{array}{ll}
h_{1}+\sigma \tau h_{3}=0, & h_{7}+\sigma \tau h_{5}=0, \\
h_{2}+\sigma \tau h_{4}=0, & h_{8}+\sigma \tau h_{6}=0,
\end{array}
$$

which are solved as

$$
\begin{aligned}
& h_{3}=-\tau h_{1}, \quad h_{7}=-\sigma \tau h_{1}^{*}, \\
& h_{4}=-\tau h_{2}, \quad h_{8}=-\sigma \tau h_{2}^{*} .
\end{aligned}
$$

Then the structure of $\hat{H}_{t}^{\prime \prime}$ can be expressed in terms of only $h_{1}, h_{2}$ and their complex conjugates as 


$$
\hat{H}_{t}^{\prime \prime}=i\left\{\alpha^{+} \beta_{t}+\tilde{\alpha}^{+} \tilde{\beta}_{t}\right\}
$$

where we introduced new operators

$$
\begin{aligned}
\alpha^{+} & =a^{\dagger}-\tau \tilde{a}, \\
\beta_{t} & =h_{1} b_{t}+h_{2} \tilde{b}_{t}^{\dagger},
\end{aligned}
$$

and their tilde conjugates. Note, that the creation operator $\alpha^{+}$annihilates the bra-vacuum $\langle\theta|$ :

$$
\langle\theta| \alpha^{+}=0 .
$$

In order to investigate parameters $h_{1}$ and $h_{2}$ we consider the moments

$$
\begin{aligned}
& \left\langle\beta_{t} \tilde{\beta}_{t}\right\rangle=\left(h_{1}+\sigma \tau h_{2}\right)\left\{\tau h_{1}^{*}\left\langle b_{t}^{\dagger} b_{t}\right\rangle+h_{2}^{*}\left\langle b_{t} b_{t}^{\dagger}\right\rangle\right\}, \\
& \left\langle\tilde{\beta}_{t} \beta_{t}\right\rangle=\left(h_{1}^{*}+\tau h_{2}^{*}\right)\left\{\sigma \tau h_{1}\left\langle b_{t}^{\dagger} b_{t}\right\rangle+h_{2}\left\langle b_{t} b_{t}^{\dagger}\right\rangle\right\},
\end{aligned}
$$

where we are using the symbol $\langle\cdots\rangle=\langle|\cdots| t\rangle$ without specifying the dynamics which determines the ket-vacuum $|t\rangle$ of the external system. For the present purpose, the details of its dynamics are not required. Here we assume, however, that the external ket-vacuum may evolve in time. The further use of the property of the (anti-)commutativity, i.e. $\left\langle\beta_{t} \tilde{\beta}_{t}\right\rangle=\sigma\left\langle\tilde{\beta}_{t} \beta_{t}\right\rangle$, gives the necessary two relations to define $h_{1}$ and $h_{2}$ :

$$
\begin{aligned}
\left(\tau h_{1}+h_{2}\right) h_{1}^{*} & =\left(\tau h_{1}^{*}+\sigma h_{2}^{*}\right) h_{1}, \\
\sigma\left(\tau h_{1}+h_{2}\right) h_{2}^{*} & =\left(\tau h_{1}^{*}+\sigma h_{2}^{*}\right) h_{2},
\end{aligned}
$$

which reduce to

$$
h_{1}^{*} h_{2}=\sigma h_{1} h_{2}^{*}
$$

We can express $h_{1}$ and $h_{2}$ as

$$
h_{1}=\mu \mathrm{e}^{i \theta_{1}}, \quad h_{2}=\nu \mathrm{e}^{i \theta_{2}}
$$

where $\mu, \nu \in \mathbf{R}$, namely $\mu=\left|h_{1}\right|, \nu=\left|h_{2}\right|$. From the requirement (5.28), one has $\theta_{2}=\theta_{1}$ for $\sigma=1$ and $\theta_{2}=\theta_{1}-\pi / 2$ for $\sigma=-1$. Substituting (5.29) into (5.22) and putting the phase factor $\mathrm{e}^{i \theta_{1}}$ into $b_{t}$ and $\tilde{b}_{t}^{\dagger}$, we have

$$
\beta_{t}=\mu b_{t}+\sigma \tau \nu \tilde{b}_{t}^{\dagger} .
$$


Thus, the vector \langle|$\beta_{t}$ is calculated as

$$
\langle| \beta_{t}=\langle|\left(\mu b_{t}+\sigma \tau \nu \tilde{b}_{t}^{\dagger}\right)=(\mu+\sigma \nu)\langle| b_{t} .
$$

The further requirement that the norm of \langle|$\beta_{t}$ should be equal to that of \langle|$b_{t}$, i.e. $\|\langle| \beta_{t}\|=\|\langle| b_{t} \|$, leads one to the relation

$$
\mu+\sigma \nu=1
$$

\subsection{Relation between the two interaction hat-Hamiltonians}

Note that the hermitian interaction hat-Hamiltonian $\hat{H}_{t}^{\prime}$ and the non-hermitian one $\hat{H}_{t}^{\prime \prime}$ are related to each other by

$$
\hat{H}_{t}^{\prime}=\hat{H}_{t}^{\prime \prime}-i\left\{\beta_{t}^{q}\left(\mu a+\sigma \tau \nu \tilde{a}^{\dagger}\right)+\tilde{\beta}_{t}^{q}\left(\mu \tilde{a}+\sigma \tau^{*} \nu a^{\dagger}\right)\right\}
$$

With an auxiliary parameter $0 \leq \lambda \leq 1$, it is possible to make a simultaneous consideration of both hermitian and non-hermitian interaction hatHamiltonians by introducing

$$
\hat{H}_{\mathrm{I}, t}=i\left\{\alpha^{+} \beta_{t}+\tilde{\alpha}^{+} \tilde{\beta}_{t}\right\}-i \lambda\left\{\beta_{t}^{+}\left(\mu a+\sigma \tau \nu \tilde{a}^{\dagger}\right)+\tilde{\beta}_{t}^{+}\left(\mu \tilde{a}+\sigma \tau^{*} \nu a^{\dagger}\right)\right\} .
$$

It is easy to see that this expression is reduced to $\hat{H}_{t}^{\prime}$ in the case $\lambda=1$ and to $\hat{H}_{t}^{\prime \prime}$ in the case $\lambda=0$, respectively. The dynamics of the system is now described by the Shrödinger equation (5.10) or (5.14) with $\hat{H}_{\mathrm{I}, t}$ in $\hat{H}_{t}^{\text {tot }}$ being given by (5.34).

\section{Quantum stochastic differential equations}

\subsection{Quantum stochastic Liouville equations}

\subsubsection{Itô type}

Let us derive the general form of the semi-free hat-Hamiltonian $\hat{\mathcal{H}}_{F, t} d t$ for a stochastic Liouville equation of the Itô type

$$
d\left|O_{F}(t)\right\rangle=-i \hat{\mathcal{H}}_{F, t} d t\left|O_{F}(t)\right\rangle
$$


where a subscript $F$ is added to indicate that we are considering a system under the influence of a random force. We assume that the hat-Hamiltonian $\hat{\mathcal{H}}_{F, t} d t$ for the stochastic semi-free field is bilinear in $a, a^{\dagger}, d F_{t}, d F_{t}^{\dagger}$ and their tilde conjugates, and that it is invariant under the phase transformation $a \rightarrow$ $a \mathrm{e}^{i \phi}$ and $d F_{t} \rightarrow d F_{t} \mathrm{e}^{i \phi}$. Here, $a, a^{\dagger}$ and their tilde conjugates are operators of a relevant system satisfying the canonical (anti-)commutation relation

$$
\left[a, a^{\dagger}\right]_{-\sigma}=1
$$

whereas $d F_{t}, d F_{t}^{\dagger}$ and their tilde conjugates are random force operators. The tilde and non-tilde operators are related with each other by the TSC

$$
\begin{aligned}
\langle\theta| \tilde{a}^{\dagger} & =\tau^{*}\langle\theta| a, \\
\langle| d \tilde{F}_{t}^{\dagger} & =\tau^{*}\langle| d F_{t},
\end{aligned}
$$

where $\langle\theta|$ and \langle| are, respectively, the thermal bra-vacuum of the relevant system and of the random force.

From the investigation in section 5, we can propose that the required form of the hat-Hamiltonian should be

$$
\hat{\mathcal{H}}_{F, t} d t=\hat{H}_{t} d t+d \hat{M}_{t}
$$

where $\hat{H}_{t}$ is specified by (3.4) with $\hat{\Pi}_{t}$ having the same structure as (3.28) or (3.51). For later convenience, we rewrite $\hat{\Pi}_{t}$ as

$$
\hat{\Pi}_{t}=\hat{\Pi}_{\mathrm{R}}+\hat{\Pi}_{\mathrm{D}}
$$

with

$$
\begin{aligned}
& \hat{\Pi}_{\mathrm{R}}=-\kappa(t)\left\{\alpha^{+} \alpha+\tilde{\alpha}^{+} \tilde{\alpha}\right\}, \\
& \hat{\Pi}_{\mathrm{D}}=\sigma \tau\{2 \kappa(t)[n(t)+\eta]+\dot{n}(t)\} \alpha^{+} \tilde{\alpha}^{q},
\end{aligned}
$$

where we introduced

$$
\alpha=\xi a+\sigma \tau \eta \tilde{a}^{\dagger}, \quad \xi+\sigma \eta=1
$$

which forms a canonical set with $\alpha^{q}$ defined by (5.21), i.e.

$$
\left[\alpha, \alpha^{\text {क }}\right]_{-\sigma}=1 .
$$


The one-particle distribution function $n(t)$ is defined by

$$
n(t)=\left\langle\left\langle\theta\left|a^{\dagger} a\right| 0_{F}(t)\right\rangle\right\rangle
$$

and satisfies the Boltzmann equation (D.11) (see Appendix D). Here, $\langle\cdots\rangle$ means to take the random average, i.e., the vacuum expectation value with respect to the thermal bra- and ket-vacuums of random force: $\langle\cdots\rangle=\langle|\cdots|\rangle$. Terms $\hat{\Pi}_{\mathrm{R}}$ and $\hat{\Pi}_{\mathrm{D}}$ are, respectively, the relaxational and diffusive parts of the damping operator $\hat{\Pi}_{t}$.

The martingale $d \hat{M}_{t}$ is the term containing operators representing quantum Brownian motion and satisfies

$$
\left\langle d \hat{M}_{t}\right\rangle=0
$$

Associating $d F_{t}$ and $d F_{t}^{\dagger}$ with $b_{t} d t$ and $b_{t}^{\dagger} d t$ in (5.34), respectively, we have

$$
\begin{aligned}
d \hat{M}_{t}= & i\left\{\alpha^{\text {q }} d W_{t}+\tilde{\alpha}^{\text {q }} d \tilde{W}_{t}\right\} \\
& -i \lambda\left\{d W_{t}^{\text {q }}\left(\mu a+\sigma \tau \nu \tilde{a}^{\dagger}\right)+d \tilde{W}_{t}^{\text {q }}\left(\mu \tilde{a}+\sigma \tau^{*} \nu a^{\dagger}\right)\right\},
\end{aligned}
$$

where we introduced new operators

$$
\begin{aligned}
d W_{t} & =\mu d F_{t}+\sigma \tau \nu d \tilde{F}_{t}^{\dagger}, \\
d W_{t}^{\text {qे }} & =d F_{t}^{\dagger}-\tau d \tilde{F}_{t} .
\end{aligned}
$$

Note that $d W_{t}^{\text {q }}$ and $d \tilde{W}_{t}^{\text {q }}$ annihilate the bra-vacuum for random force \langle| :

$$
\langle| d W_{t}^{\text {+ }}=0 .
$$

In the Itô multiplication, the random force operators $d W_{t}, d W_{t}^{\text {q }}$ and their tilde conjugates do not correlate with quantities at time $t$, e.g. $\left|O_{F}(t)\right\rangle$ :

$$
\left.\left\langle d \hat{M}_{t} \mid O_{F}(t)\right\rangle\right\rangle=0
$$

Thus, taking the random average of the stochastic Liouville equation (6.1), we arrive at the Fokker-Planck equation

$$
\frac{\partial}{\partial t}|O(t)\rangle=-i \hat{H}_{t}|O(t)\rangle
$$


where $\left.|O(t)\rangle=\left\langle\mid O_{F}(t)\right\rangle\right\rangle$.

The formal solution of (6.1) can be written as

$$
\left|O_{F}(t)\right\rangle=\hat{V}_{F}(t)\left|O_{F}(0)\right\rangle
$$

where the time-evolution generator is defined through

$$
d \hat{V}_{F}(t)=-i \hat{\mathcal{H}}_{F, t} d t \hat{V}_{F}(t)
$$

with the initial condition $\hat{V}_{F}(0)=1$.

\subsubsection{Fluctuation-dissipation theorem of the second kind}

By making use of the relation between the Itô and the Stratonovich stochastic multiplications (see Appendix A), we can rewrite the Itô type stochastic Liouville equation into the Stratonovich type as follows. Relation (A.6) makes the term containing random force operators in the r.h.s of (6.1) be

$$
d \hat{M}_{t}\left|O_{F}(t)\right\rangle=d \hat{M}_{t} \circ\left|O_{F}(t)\right\rangle-\frac{1}{2} d \hat{M}_{t} d\left|O_{F}(t)\right\rangle
$$

where the symbol o has been introduced to indicate the Stratonovich stochastic multiplication (see Appendix A). Substituting (6.1) into the last term for $d\left|O_{F}(t)\right\rangle$ and neglecting terms of the higher order than $d t$, we arrive at the quantum stochastic Liouville equation of the Stratonovich type

$$
d\left|O_{F}(t)\right\rangle=-i \hat{H}_{F, t} d t \circ\left|O_{F}(t)\right\rangle
$$

with

$$
\begin{aligned}
\hat{H}_{F, t} d t & =\hat{H}_{\mathrm{S}, t} d t+i \hat{\Pi}_{t} d t+d \hat{M}_{t}+\frac{i}{2} d \hat{M}_{t} d \hat{M}_{t} \\
& =\hat{H}_{\mathrm{S}, t} d t+i(1-\lambda) \hat{\Pi}_{\mathrm{R}} d t+d \hat{M}_{t}
\end{aligned}
$$

In order to obtain expression (6.24) we used the generalized fluctuationdissipation theorem of the second kind, which can be written as

$$
d \hat{M}_{t} d \hat{M}_{t}=-2\left(\lambda \hat{\Pi}_{\mathrm{R}}+\hat{\Pi}_{\mathrm{D}}\right) d t
$$


(refer to Appendix D for derivation). Note that in the Stratonovich multiplication random force operators $d W_{t}, d W_{t}^{\text {q }}$ and their tilde conjugates correlate with quantities at time $t$, i.e.,

$$
\left.\left\langle d \hat{M}_{t} \circ \mid O_{F}(t)\right\rangle\right\rangle \neq 0
$$

The formal solution of (6.22) has the form (6.19), where the stochastic timeevolution generator $\hat{V}_{F}(t)$ is defined through

$$
d \hat{V}_{F}(t)=-i \hat{H}_{F, t} d t \circ \hat{V}_{F}(t)
$$

with the initial condition $\hat{V}_{F}(0)=1$.

\subsubsection{Correlations of the random force operators}

Operators $d W_{t}, d W_{t}^{\text {f }}$ and their tilde conjugates are of the quantum stochastic Wiener process satisfying (for derivation of the results see Appendix D)

$$
\begin{aligned}
\left\langle d W_{t}\right\rangle & =\left\langle d \tilde{W}_{t}\right\rangle=0 \\
\left\langle d W_{t} d W_{s}\right\rangle & =\left\langle d \tilde{W}_{t} d \tilde{W}_{s}\right\rangle=0 \\
\left\langle d W_{t} d \tilde{W}_{s}\right\rangle & =\sigma\left\langle d \tilde{W}_{s} d W_{t}\right\rangle \\
& =\tau\{2 \kappa(t)[n(t)+\nu]+\dot{n}(t)\} \delta(t-s) d t d s
\end{aligned}
$$

and

$$
\begin{aligned}
\left\langle d W_{t}^{\text {q }}\right\rangle & =\left\langle d \tilde{W}_{t}^{\text {q }}\right\rangle=0, \\
\left\langle d W_{t}^{\text {q }} d W_{s}^{\text {q }}\right\rangle & =\left\langle d W_{t}^{\text {q }} d \tilde{W}_{s}^{\text {qे }}\right\rangle=0, \\
\left\langle d W_{t}^{\text {q }} d W_{s}\right\rangle & =\left\langle d \tilde{W}_{t}^{\text {q }} d \tilde{W}_{s}\right\rangle=0, \\
\left\langle d W_{t} d W_{s}^{\text {q }}\right\rangle & =\left\langle d \tilde{W}_{t} d \tilde{W}_{s}^{\text {q }}\right\rangle=2 \kappa(t) \delta(t-s) d t d s .
\end{aligned}
$$

Due to the argument of Appendix D we also have $\xi=\mu$ and $\eta=\nu$, which leads to

$$
\alpha=\mu a+\sigma \tau \nu \tilde{a}^{\dagger}
$$

In the following, we will use this definition in both $\hat{\Pi}_{t}$ and $d \hat{M}_{t}$. Especially, the latter becomes

$$
d \hat{M}_{t}=i\left\{\alpha^{+} d W_{t}+\tilde{\alpha}^{\text {q }} d \tilde{W}_{t}\right\}-i \lambda\left\{d W_{t}^{\text {q }} \alpha+d \tilde{W}_{t}^{\text {q }} \tilde{\alpha}\right\} .
$$


It is important to note here that the martingale $d \hat{M}_{t}$ is introduced in the normal ordering with respect to all operators $\alpha^{+}, \alpha, d W_{t}^{+}, d W_{t}$ and their tilde conjugates.

Within the weak relations, the correlations (6.29), (6.30) and (6.32) to (6.34) reduce, respectively, to

$$
\begin{aligned}
& d W_{t} d W_{s}=d \tilde{W}_{t} d \tilde{W}_{s}=0 \\
& d W_{t} d \tilde{W}_{s}=\sigma d \tilde{W}_{s} d W_{t}=\tau\{2 \kappa(t)[n(t)+\nu]+\dot{n}(t)\} \delta(t-s) d s d t
\end{aligned}
$$

and

$$
\begin{aligned}
d W_{t}^{\text {q }} d W_{s}^{\text {q }} & =d W_{t}^{\text {q }} d \tilde{W}_{s}^{\text {q }}=0, \\
d W_{t}^{\text {q }} d W_{s} & =d \tilde{W}_{t}^{\text {q }} d \tilde{W}_{s}=0, \\
d W_{t} d W_{s}^{\text {क }} & =d \tilde{W}_{t} d \tilde{W}_{s}^{\text {क }}=2 \kappa(t) \delta(t-s) d s d t .
\end{aligned}
$$

\subsection{Stochastic semi-free operators}

The stochastic semi-free operators are defined by

$$
A(t)=\hat{V}_{F}^{-1}(t) A \hat{V}_{F}(t)
$$

whereas the random force operators in the Heisenberg representation by

$$
W(t)=\hat{V}_{F}^{-1}(t) W_{t} \hat{V}_{F}(t), \quad W^{\text {q }}(t)=\hat{V}_{F}^{-1}(t) W_{t}^{\text {甲 }} \hat{V}_{F}(t),
$$

and their tilde conjugates. We also use the convenient operators introduced by

$$
\vec{d} W(t)=\hat{V}_{F}^{-1}(t) d W_{t} \hat{V}_{F}(t), \vec{d} W^{\text {q }}(t)=\hat{V}_{F}^{-1}(t) d W_{t}^{\text {q }} \hat{V}_{F}(t),
$$

and their tilde conjugates. Here,

$$
d \hat{V}_{F}^{-1}(t)=i \hat{V}_{F}^{-1}(t) \hat{\mathcal{H}}_{F, t}^{-} d t
$$

with $\hat{V}_{F}^{-1}(0)=1 . \hat{\mathcal{H}}_{F, t}^{-} d t$ is specified by

$$
\hat{\mathcal{H}}_{F, t}^{-} d t=\hat{\mathcal{H}}_{F, t} d t+i d \hat{M}_{t} d \hat{M}_{t}=\hat{H}_{\mathrm{S}, t} d t+i \hat{\Pi}_{t}^{-} d t+d \hat{M}_{t}
$$


with

$$
\hat{\Pi}_{t}^{-}=(1-2 \lambda) \hat{\Pi}_{\mathrm{R}}-\hat{\Pi}_{\mathrm{D}}
$$

In particular cases when $A$ represents $a$ or $a^{\dagger}$ we have

$$
a(t)=\hat{V}_{F}^{-1}(t) a \hat{V}_{F}(t), \quad \tilde{a}^{\dagger}(t)=\hat{V}_{F}^{-1}(t) \tilde{a}^{\dagger} \hat{V}_{F}(t)
$$

Since the stochastic tildian hat-Hamiltonian $\hat{\mathcal{H}}_{F, t} d t$ is not necessarily hermitian, we introduced the symbol + in order to distinguish it from the hermite conjugation $\dagger$. It is assumed that, at initial time $t=0$, the relevant system starts to contact with the irrelevant system representing the stochastic process described by the random force operators $d F_{t}, d F_{t}^{\dagger}$ and their tilde conjugates. Within the formalism, the random force operators $d F_{t}$ and $d F_{t}^{\dagger}$ are assumed to (anti-)commute with any relevant system operator $A$ in the Schrödinger representation, i.e.

$$
\left[A, d F_{t}\right\}=0, \quad\left[A, d F_{t}^{\dagger}\right\}=0 .
$$

The semi-free operators $a(t), a^{\dagger}(t)$ and their tilde conjugates keep the equaltime canonical (anti-)commutation relations

$$
\left[a(t), a^{\dagger}(t)\right]_{-\sigma}=1
$$

and satisfy TSC

$$
\left\langle\langle\theta| \tilde{a}^{\dagger \dagger}(t)=\tau^{*}\langle\langle\theta| a(t) .\right.
$$

Calculating the time derivatives of Heisenberg operators of the quantum Brownian motion (6.43) within the Itô calculus (A.15), and taking into account (6.20), (6.45) with the characteristics of the Itô multiplication

$$
\begin{aligned}
{\left[d W_{t}^{\text {q }}, W_{t}\right]_{-\sigma} } & =\left[d W_{t}, W_{t}^{\text {q }}\right]_{-\sigma}=0, \\
{\left[d W_{t}, \hat{V}_{F}(t)\right] } & =\left[d W_{t}^{\text {q }}, \hat{V}_{F}(t)\right]=0,
\end{aligned}
$$

and their tilde conjugates, one has

$$
\begin{gathered}
d W(t)=d W_{t}-i \hat{V}_{F}^{-1}(t)\left[d W_{t}, d \hat{M}_{t}\right] \hat{V}_{F}(t), \\
d W^{\text {क }}(t)=d W_{t}^{\text {+ }}-i \hat{V}_{F}^{-1}(t)\left[d W_{t}^{\text {q }}, d \hat{M}_{t}\right] \hat{V}_{F}(t),
\end{gathered}
$$


and their tilde conjugates. With the help of (6.37) to (6.41), the expressions (6.54) and (6.55) reduce, respectively, to

$$
\begin{aligned}
d W(t) & =d W_{t}-2 \lambda \kappa(t) \alpha(t) d t, \\
d W^{\text {q }}(t) & =d W_{t}^{\text {क }}-2 \kappa(t) \alpha^{\text {क }}(t) d t,
\end{aligned}
$$

(and their tilde conjugates), while (6.53) gives

$$
\vec{d} W(t)=d W_{t}, \quad \vec{d} W^{\text {q }}(t)=d W_{t}^{\text {q }},
$$

(and their tilde conjugates).

We see that the martingale operator $\vec{d} \hat{M}(t) \equiv \hat{V}_{F}^{-1}(t) d \hat{M}_{t} \hat{V}_{F}(t)$ being written in terms of the Heisenberg operators reads

$$
\begin{aligned}
\vec{d} \hat{M}(t)= & i\left\{\alpha^{\text {}}(t) \vec{d} W(t)+\tilde{\alpha}^{\text {甲 }}(t) \vec{d} \tilde{W}(t)\right\} \\
& -i \lambda\left\{\vec{d} W^{\text {क }}(t) \alpha(t)+\vec{d} \tilde{W}^{\text {क }}(t) \tilde{\alpha}(t)\right\} \\
= & i\left\{\alpha^{\text {甲 }}(t) d W(t)+\tilde{\alpha}^{\text {甲 }}(t) d \tilde{W}(t)\right\} \\
& -i \lambda\left\{d W^{\text {क }}(t) \alpha(t)+d \tilde{W}^{\text {क }}(t) \tilde{\alpha}(t)\right\} \\
\equiv & d \hat{M}(t),
\end{aligned}
$$

and keeps the property

$$
\langle\vec{d} \hat{M}(t)\rangle=\langle d \hat{M}(t)\rangle=0
$$

for arbitrary $\lambda$. The beautiful relation (6.59) is manifestations of the normal ordered definition (6.36). Note that the increments in the martingale (6.36) are introduced just through the random force operators $d W_{t}^{q}, d W_{t}$ and their tilde conjugates. Therefore, $d \hat{M}(t)$ is different from the operator calculated by $d\left(i\left\{\alpha^{+}(t) W(t)+\right.\right.$ t.c. $\}-i \lambda\left\{W^{q}(t) \alpha(t)+\right.$ t.c. $\left.\}\right)$. Here, t.c. indicates the tilde conjugation.

\subsection{Quantum Langevin equations}

\subsubsection{Itô type}

Substituting $d \hat{V}_{F}(t),(6.20)$, and $d \hat{V}_{F}^{-1}(t),(6.45)$, into the time derivative of the dynamical quantity $A(t)$ within the Itô calculus (A.15), we obtain the quantum Langevin equation of the Itô type in the form 


$$
\begin{aligned}
& d A(t)=i\left[\hat{\mathcal{H}}_{F}(t) d t, A(t)\right]-d \hat{M}(t)[d \hat{M}(t), A(t)] \\
& =i\left[\hat{H}_{\mathrm{S}}(t), A(t)\right] d t \\
& +\kappa(t)\left(\alpha^{q}(t)[\alpha(t), A(t)\}+\tilde{\alpha}^{q}(t)[\tilde{\alpha}(t), A(t)\}\right. \\
& \left.+(2 \lambda-1)\left(\left[A(t), \alpha^{+}(t)\right\} \alpha(t)+\left[A(t), \tilde{\alpha}^{+}(t)\right\} \tilde{\alpha}(t)\right)\right) d t \\
& +\tau(2 \kappa(t)[n(t)+\nu]+\dot{n}(t))\left[\tilde{\alpha}^{+}(t),\left[\alpha^{+}(t), A(t)\right\}\right\} d t \\
& +\left[A(t), \alpha^{+}(t)\right\} d W(t)+\left[A(t), \tilde{\alpha}^{+}(t)\right\} d \tilde{W}(t) \\
& +\lambda\left(d W^{\text {f }}(t)[\alpha(t), A(t)\}+d \tilde{W}^{\text {क }}(t)[\tilde{\alpha}(t), A(t)\}\right) \\
& =i\left[\hat{H}_{\mathrm{S}}(t), A(t)\right] d t \\
& -\kappa(t)\left(\left[A(t), \alpha^{+}(t)\right\} \alpha(t)+\left[A(t), \tilde{\alpha}^{q}(t)\right\} \tilde{\alpha}(t)\right. \\
& \left.+(2 \lambda-1)\left(\alpha^{+}(t)[\alpha(t), A(t)\}+\tilde{\alpha}^{+}(t)[\tilde{\alpha}(t), A(t)\}\right)\right) d t \\
& +\tau(2 \kappa(t)[n(t)+\nu]+\dot{n}(t))\left[\tilde{\alpha}^{q}(t),\left[\alpha^{+}(t), A(t)\right\}\right\} d t \\
& +\left[A(t), \alpha^{\text {q }}(t)\right\} d W_{t}+\left[A(t), \tilde{\alpha}^{q}(t)\right\} d \tilde{W}_{t} \\
& +\lambda\left(d W_{t}^{\text {q }}[\alpha(t), A(t)\}+d \tilde{W}_{t}^{\text {क }}[\tilde{\alpha}(t), A(t)\}\right),
\end{aligned}
$$

where (6.37) to (6.41) for multiplications among the random force operators are employed. To derive (6.63) from (6.62), we used (6.56) and (6.57). Note that the Langevin equations (6.62) and (6.63) written, respectively, by means of the quantum Brownian motion in the Heisenberg representation and by means of that in the Schrödinger representation may be related with the "out" and "in" fields introduced by Gardiner et al. [77,78].

With the help of (6.62) one can verify that the calculus rule for the product of arbitrary relevant stochastic operators, say $A(t)$ and $B(t)$, satisfies the Itô calculus (A.15). This proves that QSDE (6.62) is of the Itô type indeed. Furthermore, since (6.62) is the time-evolution equation for any relevant stochastic operator $A(t)$, it is Itô's formula for quantum systems.

\subsubsection{Stratonovich type}

The quantum Langevin equation of the Stratonovich type can be derived similarly if one starts from the expression for a dynamical quantity $A(t),(6.42)$, and considers its derivative in the Stratonovich calculus (A.16) with the help of

$$
d \hat{V}_{F}^{-1}(t)=i \hat{V}_{F}^{-1}(t) \circ \hat{H}_{F, t} d t
$$

Substituting (6.27) for $d \hat{V}_{F}(t)$ and (6.64) for $d \hat{V}_{F}^{-1}(t)$ into $d A(t)$, we have for the stochastic Heisenberg equation of the Stratonovich type 


$$
\begin{aligned}
& d A(t)=i\left[\hat{H}_{F}(t) d t ; A(t)\right] \\
& =i\left[\hat{H}_{\mathrm{S}}(t), A(t)\right] d t \\
& +\kappa(t)\left(\alpha^{+}(t)[\alpha(t), A(t)\}+\tilde{\alpha}^{+}(t)[\tilde{\alpha}(t), A(t)\}\right. \\
& \left.+(2 \lambda-1)\left(\left[A(t), \alpha^{+}(t)\right\} \alpha(t)+\left[A(t), \tilde{\alpha}^{+}(t)\right\} \tilde{\alpha}(t)\right)\right) d t \\
& +\left[A(t), \alpha^{+}(t)\right\} \circ d W(t)+\left[A(t), \tilde{\alpha}^{+}(t)\right\} \circ d \tilde{W}(t) \\
& +\lambda\left(d W^{\text {q }}(t) \circ[\alpha(t), A(t)\}+d \tilde{W}^{\text {क }}(t) \circ[\tilde{\alpha}(t), A(t)\}\right) \\
& =i\left[\hat{H}_{\mathrm{S}}(t), A(t)\right] d t \\
& -\kappa(t)\left(\left[A(t), \alpha^{q}(t)\right\} \alpha(t)+\left[A(t), \tilde{\alpha}^{+}(t)\right\} \tilde{\alpha}(t)\right. \\
& \left.+(2 \lambda-1)\left(\alpha^{+}(t)[\alpha(t), A(t)\}+\tilde{\alpha}^{+}(t)[\tilde{\alpha}(t), A(t)\}\right)\right) d t \\
& +\left[A(t), \alpha^{+}(t)\right\} \circ d W_{t}+\left[A(t), \tilde{\alpha}^{+}(t)\right\} \circ d \tilde{W}_{t} \\
& +\lambda\left(d W_{t}^{\text {q }} \circ[\alpha(t), A(t)\}+d \tilde{W}_{t}^{\text {q }} \circ[\tilde{\alpha}(t), A(t)\}\right) \text {. }
\end{aligned}
$$

Here, we defined

$$
[X(t) \stackrel{\circ}{\prime}(t)]=X(t) \circ Y(t)-Y(t) \circ X(t)
$$

for arbitrary operators $X(t)$ and $Y(t)$, and

$$
\hat{H}_{F}(t) d t=\hat{V}_{F}^{-1}(t) \circ \hat{H}_{F, t} d t \circ \hat{V}_{F}(t) .
$$

Note that

$$
\begin{aligned}
\hat{V}_{F}^{-1}(t) \circ d \hat{M}_{t} \circ \hat{V}_{F}(t)= & \hat{V}_{F}^{-1}(t) d \hat{M}_{t} \hat{V}_{F}(t) \\
& +\frac{1}{2} \hat{V}_{F}^{-1}(t) d \hat{M}_{t} d \hat{V}_{F}(t)+\frac{1}{2} d \hat{V}_{F}^{-1}(t) d \hat{M}_{t} \hat{V}_{F}(t) \\
= & d \hat{M}(t) .
\end{aligned}
$$

Using expression (6.66), one can readily verify that the calculus rule for the product of arbitrary relevant system operators, say $A(t)$ and $B(t)$, satisfies the Stratonovich type calculus (A.16). This fact proves that QSDE (6.66) is indeed of the Stratonovich type, and provides us with the reason why the stochastic Heisenberg equation (6.65) has the same structure as the one (2.14) for non-stochastic operators.

The quantum Langevin equation of the Stratonovich type can be also derived from that of the Itô type by making use the connection formulae (A.13) and (A.14). When $d Y(t)$ is $d W(t)$ or $d \tilde{W}(t)$, and $X(t)$ is constituted by the relevant operator, say $A(t)$, satisfying the quantum Langevin equation of the Itô type, the connection formula (A.13) reduces, respectively, to 


$$
\begin{aligned}
A(t) \cdot d W(t)= & A(t) \circ d W(t) \\
& -\frac{1}{2} \sigma \tau(2 \kappa(t)[n(t)+\nu]+\dot{n}(t))\left[A(t), \tilde{\alpha}^{+}(t)\right\} d t, \\
A(t) \cdot d \tilde{W}(t)= & A(t) \circ d \tilde{W}(t) \\
& -\frac{1}{2} \tau(2 \kappa(t)[n(t)+\nu]+\dot{n}(t))\left[A(t), \alpha^{q}(t)\right\} d t .
\end{aligned}
$$

Similarly, when $d X(t)$ is $d W^{\text {q }}(t)$ or $d \tilde{W}^{\text {f }}(t)$, and $Y(t)$ is $A(t)$, the connection formula (A.14) reduces, respectively, to

$$
\begin{aligned}
& d W^{\text {q }}(t) \cdot A(t)=d W^{\text {q }}(t) \circ A(t), \\
& d \tilde{W}^{\text {f }}(t) \cdot A(t)=d \tilde{W}^{\text {क }}(t) \circ A(t) .
\end{aligned}
$$

Using these relations in (6.62), the quantum Langevin equation of the Stratonovich type is obtained in the form (6.66).

Substituting $\alpha$ and $\tilde{\alpha}^{\text {q }}$ for $A$ as an example, we see that both (6.61) and (6.65) result in

$$
\begin{aligned}
d \alpha(t) & =-i \omega(t) \alpha(t) d t-(1-2 \lambda) \kappa(t) \alpha(t) d t+d W(t) \\
& =-[i \omega(t)+\kappa(t)] \alpha(t) d t+d W_{t}, \\
d \tilde{\alpha}^{q}(t) & =-i \omega(t) \tilde{\alpha}^{q}(t) d t+\kappa(t) \tilde{\alpha}^{q}(t) d t+\lambda d \tilde{W}^{\text {q }}(t) \\
& =-[i \omega(t)-(1-2 \lambda) \kappa(t)] \tilde{\alpha}^{q}(t) d t+\lambda d \tilde{W}_{t}^{+},
\end{aligned}
$$

which are written in terms of the original operators as

$$
\begin{aligned}
d a(t)= & -[i \omega(t)-\lambda \kappa(t)] a(t) d t \\
& -(1-\lambda) \kappa(t)\left[(\mu-\sigma \nu) a(t)+2 \sigma \tau \nu \tilde{a}^{\dagger}(t)\right] d t \\
& +d W(t)-\lambda \sigma \tau \nu d \tilde{W}^{\text {क }}(t) \\
= & -[i \omega(t)+\lambda \kappa(t)] a(t) d t \\
& -(1-\lambda) \kappa(t)\left[(\mu-\sigma \nu) a(t)+2 \sigma \tau \nu \tilde{a}^{\dagger}(t)\right] d t \\
& +d W_{t}-\lambda \sigma \tau \nu d \tilde{W}_{t}^{\text {q }} \\
d \tilde{a}^{\dagger}(t)=- & {[i \omega(t)-\lambda \kappa(t)] \tilde{a}^{\dagger}(t) d t } \\
& +(1-\lambda) \kappa(t)\left[(\mu-\sigma \nu) \tilde{a}^{\dagger}(t)-2 \sigma \tau \mu a(t)\right] d t \\
& +\sigma \tau d W(t)+\lambda \mu d \tilde{W}^{\text {क }}(t) \\
= & -[i \omega(t)+\lambda \kappa(t)] \tilde{a}^{\dagger}(t) d t \\
& +(1-\lambda) \kappa(t)\left[(\mu-\sigma \nu) \tilde{a}^{\dagger}(t)-2 \sigma \tau \mu a(t)\right] d t \\
& +\sigma \tau d W_{t}+\lambda \mu d \tilde{W}_{t}^{\text {q }} .
\end{aligned}
$$

These equations are the same in both Itô and Stratonovich multiplications as they should be with the martingale (6.36). 


\subsection{Averaged equation of motion}

Applying the total bra-vacuum $\langle\langle\theta|$ to the Itô type quantum Langevin equation (6.61) to (6.63), one can derive the stochastic equation of motion of the Itô type for the bra-vector state $\langle\langle\theta| A(t)$ in the form

$$
\begin{aligned}
& d\left\langle\langle\theta| A(t)=i\left\langle\langle\theta|\left[\hat{H}_{\mathrm{S}}(t), A(t)\right] d t\right.\right. \\
& +(2 \lambda-1) \kappa(t)\left\langle\langle\theta|\left(A(t)\left[\alpha^{+}(t) \alpha(t)+\tilde{\alpha}^{+}(t) \tilde{\alpha}(t)\right]\right) d t\right. \\
& +\sigma \tau(2 \kappa(t)[n(t)+\nu]+\dot{n}(t))\left\langle\langle\theta| A(t) \alpha^{+}(t) \tilde{\alpha}^{+}(t) d t\right. \\
& +\left\langle\langle\theta|\left(A(t)\left[\alpha^{+}(t) d W(t)+\tilde{\alpha}^{+}(t) d \tilde{W}(t)\right]\right)\right. \\
& =i\left\langle\langle\theta|\left[\hat{H}_{\mathrm{S}}(t), A(t)\right] d t\right. \\
& -\kappa(t)\left\langle\langle\theta|\left(A(t)\left[\alpha^{+}(t) \alpha(t)+\tilde{\alpha}^{\text {f }}(t) \tilde{\alpha}(t)\right]\right) d t\right. \\
& +\sigma \tau(2 \kappa(t)[n(t)+\nu]+\dot{n}(t))\left\langle\langle\theta| A(t) \alpha^{+}(t) \tilde{\alpha}^{+}(t) d t\right. \\
& +\left\langle\langle\theta|\left(A(t)\left[\alpha^{+}(t) d W_{t}+\tilde{\alpha}^{\text {f }}(t) d \tilde{W}_{t}\right]\right)\right. \text {. }
\end{aligned}
$$

In terms of operators $a(t)$ and $a^{\dagger}(t)$ it becomes

$$
\begin{aligned}
d\langle\langle\theta| A(t)= & i\left\langle\langle\theta|\left[\hat{H}_{\mathrm{S}}(t), A(t)\right] d t\right. \\
& -\kappa(t)\left\langle\langle\theta|\left(\left[A(t), a^{\dagger}(t)\right\} a(t)+a^{\dagger}(t)[a(t), A(t)\}\right) d t\right. \\
& -\sigma(2 \kappa(t) n(t)+\dot{n}(t))\left\langle\langle\theta|\left[\left[A(t), a^{\dagger}(t)\right\}, a(t)\right\} d t\right. \\
& +\left\langle\langle\theta|\left(\left[A(t), a^{\dagger}(t)\right\} d F_{t}-\sigma[A(t), a(t)\} d F_{t}^{\dagger}\right),\right.
\end{aligned}
$$

where we used

$$
\langle| d W_{t}=\langle| d F_{t}, \quad\langle| d \tilde{W}_{t}=\tau^{*}\langle| d F_{t}^{\dagger}
$$

The stochastic equation of motion of the Stratonovich type for the bra-vector state $\langle\langle\theta| A(t)$ is derived similarly in the form

$$
\begin{aligned}
d\langle\langle\theta| A(t)= & i\left\langle\langle\theta|\left[\hat{H}_{\mathrm{S}}(t), A(t)\right] d t\right. \\
& -\kappa(t)\left\langle\langle\theta|\left(a^{\dagger}(t)[a(t), A(t)\}-\sigma a(t)\left[a^{\dagger}(t), A(t)\right\}\right) d t\right. \\
& +\left\langle\langle\theta|\left(\left[A(t), a^{\dagger}(t)\right\} \circ d F_{t}-\sigma[A(t), a(t)\} \circ d F_{t}^{\dagger}\right) .\right.
\end{aligned}
$$

Applying to (6.81) the random force ket-vacuum |\rangle and the ket-vacuum $|0\rangle$ of the relevant system, one obtains the equation of motion for the expectation value of an arbitrary operator $A(t)$ of the relevant system as 


$$
\begin{aligned}
\frac{d}{d t}\langle\langle A(t)\rangle\rangle= & i\left\langle\left\langle\left[\hat{H}_{\mathrm{S}}(t), A(t)\right]\right\rangle\right\rangle \\
& -\kappa(t)\left\langle\left\langle\left(\left[A(t), a^{\dagger}(t)\right\} a(t)+a^{\#}(t)[a(t), A(t)\}\right)\right\rangle\right\rangle \\
& -\sigma(2 \kappa(t) n(t)+\dot{n}(t))\left\langle\left\langle\left[\left[A(t), a^{\dagger}(t)\right\}, a(t)\right\}\right\rangle\right\rangle .
\end{aligned}
$$

Here, $\langle\langle\cdots\rangle\rangle=\langle|\langle\theta|\cdots| 0\rangle|\rangle$ means to take both random average and vacuum expectation. This is the exact equation of motion for systems with lineardissipative coupling to reservoir, which can be also derived by means of the Fokker-Planck equation (6.18). Here, we used the property

$$
\left\langle\left[A(t), a^{\dagger}(t)\right\} d F_{t}\right\rangle=\left\langle[A(t), a(t)\} d F_{t}^{\dagger}\right\rangle=0,
$$

which is the characteristics of the Itô multiplication. Note that equation of motion for expectation value of an arbitrary operator $A(t)$ does not depend on the parameter $\lambda$.

\section{Semi-free system with a stationary process}

One possible way to specify a model is to give the Boltzmann equation (3.23). For the cases of a semi-free system corresponding to the stationary quantum stochastic processes, one needs to make substitutions

$$
i \Sigma^{<}(t)=2 \kappa \bar{n}, \quad \omega(t)=\omega, \quad \kappa(t)=\kappa,
$$

where $\bar{n}$ is an average quantum number in equilibrium given by

$$
\bar{n}=\left(\mathrm{e}^{\omega / T}-\sigma\right)^{-1}
$$

and $T$ is the temperature of environment (here we use the system with the Boltzmann constant $k_{\mathrm{B}}=1$ ). Then, the Boltzmann equation (3.23) becomes

$$
\dot{n}(t)=-2 \kappa(n(t)-\bar{n}) .
$$

It describes the system of a damped harmonic oscillator.

Substituting the Boltzmann equation (7.3) into the semi-free hat-Hamiltonian (3.4) with (3.5) and (3.28) or with (3.50) and (3.51), one obtains

$$
\hat{H}=\omega\left(a^{\dagger} a-\tilde{a}^{\dagger} \tilde{a}\right)+2 \sigma \tau i \kappa(1+\sigma \bar{n}) a \tilde{a}+2 \sigma \tau i \kappa \bar{n} a^{\dagger} \tilde{a}^{\dagger}
$$




$$
\begin{aligned}
& -i \kappa(1+2 \sigma \bar{n})\left(a^{\dagger} a+\tilde{a}^{\dagger} \tilde{a}\right)-2 i \kappa \bar{n} \\
= & \omega \bar{a}^{\mu} a^{\mu}-i \kappa \bar{a}^{\mu} A^{\mu \nu} a^{\nu}+\sigma(\omega+i \kappa) \\
= & \omega\left(\gamma^{\text {q }} \gamma_{t}-\tilde{\gamma}^{\text {q }} \tilde{\gamma}_{t}\right)-i \kappa\left(\gamma^{\text {q }} \gamma_{t}+\tilde{\gamma}^{\text {q }} \tilde{\gamma}_{t}\right)-2 \sigma \tau i \kappa(n(t)-\bar{n}) \gamma^{\text {q }} \tilde{\gamma}^{\text {q }},
\end{aligned}
$$

where

$$
A^{\mu \nu}=\left(\begin{array}{rr}
1+2 \sigma \bar{n} & -2 \sigma \bar{n} \\
2(1+\sigma \bar{n}) & -(1+2 \sigma \bar{n})
\end{array}\right) .
$$

The Fokker-Planck equation of the model is given by

$$
\frac{\partial}{\partial t}|0(t)\rangle=-i \hat{H}|0(t)\rangle
$$

with (7.6). It is solved as (3.53) with the order parameter

$$
\left\langle\theta\left|\tilde{\gamma}_{t} \gamma_{t}\right| 0\right\rangle=\sigma \tau(n(0)-\bar{n})\left(1-\mathrm{e}^{-2 \kappa t}\right),
$$

where $\gamma_{t}, \gamma^{q}$ and their tilde conjugates are defined by (3.47) and (3.40) with $n(t)$ being replaced by the solution of (7.3). The expression (3.53) with the order parameter (7.9) led us to the notion of a mechanism named the spontaneous creation of dissipation $[79,80,81,82,83,84]$.

Introducing a set of new operators

$$
\bar{d}^{\nu}=\left(d^{\dagger},-\tau \tilde{d}\right), \quad d^{\mu}=\operatorname{collon}\left(d, \tau \tilde{d}^{\dagger}\right),
$$

defined by

$$
\bar{d}^{\nu}=\bar{a}^{\mu}\left[B^{\mu \nu}\right]^{-1}, \quad d^{\mu}=B^{\mu \nu} a^{\nu},
$$

with

$$
B^{\mu \nu}=\left(\begin{array}{rr}
1+\sigma \bar{n} & -\sigma \bar{n} \\
-1 & 1
\end{array}\right),
$$

the hat-Hamiltonian $\hat{H}$ can be also written in the form

$$
\hat{H}=\omega\left(d^{\dagger} d-\tilde{d}^{\dagger} \tilde{d}\right)-i \kappa\left(d^{\dagger} d+\tilde{d}^{\dagger} \tilde{d}\right)
$$


We see that the new operators satisfy the canonical (anti-)commutation relation

$$
\left[d^{\mu}, \bar{d}^{\nu}\right]_{-\sigma}=\delta^{\mu \nu}
$$

and that TSC (3.24) for the thermal ket-vacuum $|0\rangle$ can be expressed as

$$
\tilde{d}|0\rangle=\tau(n(0)-\bar{n}) d^{\dagger}|0\rangle
$$

It is easy to see from the diagonalized form (7.13) of $\hat{H}$ that

$$
\begin{aligned}
d(t) & =\hat{V}^{-1}(t) d \hat{V}(t)=d \mathrm{e}^{-(i \omega+\kappa) t} \\
\tilde{d}^{\dagger}(t) & =\hat{V}^{-1}(t) \tilde{d}^{\dagger} \hat{V}(t)=\tilde{d}^{\dagger} \mathrm{e}^{-(i \omega-\kappa) t}
\end{aligned}
$$

On the other hand, it is easy to see from the normal ordered form (7.6) that $\hat{H}$ satisfies $\langle\theta| \hat{H}=0$, since the annihilation and creation operators satisfy (3.48) and (3.49). The difference between the operators which diagonalize $\hat{H}$ and the ones which make $\hat{H}$ in the form of normal product is one of the features of NETFD, and shows the point that the formalism is different from usual quantum mechanics and quantum field theory. This is manifestations of the fact that the hat-Hamiltonian is a time-evolution generator for irreversible processes.

The second law of thermodynamics tells us that for a closed system the entropy increment $d \mathcal{S}$ of the relevant system should be given by [85]

$$
\begin{aligned}
& d \mathcal{S}=d \mathcal{S}_{\mathrm{i}}+d \mathcal{S}_{\mathrm{e}} \\
& d \mathcal{S}_{\mathrm{i}} \geq 0
\end{aligned}
$$

where $d \mathcal{S}_{\mathrm{i}}$ is the change of intrinsic entropy of the system, and $d \mathcal{S}_{\mathrm{e}}$ the change due to the heat flow $\vec{d} Q$ into the system from the thermal reservoir with temperature $T$ :

$$
d \mathcal{S}_{\mathrm{e}}=\frac{\vec{a} Q}{T}
$$

We can check this for the present model [1]. The entropy of the relevant system is given by [86]

$$
\mathcal{S}(t)=-\{n(t) \ln n(t)-\sigma[1+\sigma n(t)] \ln [1+\sigma n(t)]\}
$$


whereas the heat change of the system can be identified with

$$
\vec{d} Q(t)=\omega d n(t)
$$

leading to

$$
d \mathcal{S}_{\mathrm{e}}=\frac{\omega}{T} d n(t)
$$

Putting (7.21) and (7.23) into (7.18) for $d \mathcal{S}$ and $d \mathcal{S}_{\mathrm{e}}$, respectively, we have a relation for the entropy production rate $[1]$

$$
\frac{d \mathcal{S}_{\mathrm{i}}}{d t}=\frac{d \mathcal{S}}{d t}-\frac{d \mathcal{S}_{\mathrm{e}}}{d t}=2 \kappa(n(t)-\bar{n}) \ln \frac{n(t)(1+\sigma \bar{n})}{\bar{n}(1+\sigma n(t))} \geq 0
$$

giving the inequality (7.19). It is easy to check that inequality holds for both cases, i.e. $n(t)>\bar{n}$ and $n(t)<\bar{n}$, and that the equality realizes for the thermal equilibrium state, $n(t)=\bar{n}$, or for the quasi-static process with $\kappa \rightarrow 0$.

\section{Relation to the Monte Carlo wave-function method}

In this section, we will investigate the Fokker-Planck equation (7.8) in order to reveal the relation of NETFD to the Monte Carlo wave-function method, i.e. the quantum jump simulation $[87,88,89,90,91,92,93,94,9]$ in which evolution with a non-hermitian hat-Hamiltonian is described in terms of randomly decided quantum jumps followed by the wave-function normalization.

Let us decompose the hat-Hamiltonian (7.4) as

$$
\hat{H}=\hat{H}^{(0)}+\hat{H}^{(1)},
$$

with

$$
\begin{aligned}
& \hat{H}^{(0)}=\omega\left(a^{\dagger} a-\tilde{a}^{\dagger} \tilde{a}\right)-i \kappa(1+2 \sigma \bar{n})\left(a^{\dagger} a+\tilde{a}^{\dagger} \tilde{a}\right), \\
& \hat{H}^{(1)}=2 i \sigma \tau \kappa\left((1+\sigma \bar{n}) a \tilde{a}+\bar{n} a^{\dagger} \tilde{a}^{\dagger}\right)-2 i \kappa \bar{n},
\end{aligned}
$$

and consider an equation:

$$
\frac{\partial}{\partial t}\left|0_{0}(t)\right\rangle^{\prime}=-i \hat{H}^{(0)}\left|0_{0}(t)\right\rangle^{\prime} .
$$


Note that $\hat{H}^{(1)}$ contains cross terms among tilde and non-tilde operators. We see that $\hat{H}^{(0)}$ and $\hat{H}^{(1)}$ have the properties

$$
\begin{aligned}
& \langle\theta| \hat{H}^{(0)}=-2 i \kappa(1+2 \sigma \bar{n})\langle\theta| a^{\dagger} a, \\
& \langle\theta| \hat{H}^{(1)}=2 i \kappa(1+2 \sigma \bar{n})\langle\theta| a^{\dagger} a .
\end{aligned}
$$

Introducing the wave-functions $|\psi(t)\rangle$ and $|\tilde{\psi}(t)\rangle$ through the relation

$$
\left|0_{0}(t)\right\rangle^{\prime}=|\psi(t)\rangle|\tilde{\psi}(t)\rangle
$$

we have from (8.4) the Schrödinger equations of the form

$$
\frac{\partial}{\partial t}|\psi(t)\rangle=-i H^{(0)}|\psi(t)\rangle
$$

and its tilde conjugate, where

$$
H^{(0)}=\omega a^{\dagger} a-i \kappa(1+2 \sigma \bar{n}) a^{\dagger} a .
$$

This procedure is possible because $\hat{H}^{(0)}$ does not contain cross terms among tilde and non-tilde operators. The Monte Carlo simulations for quantum systems are performed for the Schrödinger equation (8.8) [87,88,89,90].

The time evolution generated by the hat-Hamiltonian $\hat{H}^{(0)}$ does not preserve the normalization of the ket-vacuum, i.e. the normalized ket-vacuum $|0(t)\rangle$ evolves for the time increment $d t$ as

$$
\left\langle\theta \mid 0_{0}(t+d t)\right\rangle^{\prime}=\left\langle\theta\left|\left(1-i \hat{H}^{(0)} d t\right)\right| 0(t)\right\rangle=1-d p(t)
$$

with

$$
d p(t)=i\left\langle\theta\left|\hat{H}^{(0)}\right| 0(t)\right\rangle d t=2 \kappa(1+2 \sigma \bar{n}) n(t) d t
$$

The recipe of the quantum jump simulation is that, for a time increment $d t$,

1) when $d p(t)<\varepsilon$ with a given positive constant $\varepsilon$, the normalized ketvacuum evolves as

$$
|0(t)\rangle \rightarrow\left|0_{0}(t+d t)\right\rangle=\frac{\left|O_{0}(t+d t)\right\rangle^{\prime}}{1-d p(t)}
$$




$$
=\frac{|\psi(t+d t)\rangle}{\sqrt{1-d p(t)}} \frac{|\tilde{\psi}(t+d t)\rangle}{\sqrt{1-d p(t)}}
$$

2 ) in the case $d p(t)>\varepsilon$, a quantum jump comes in

$$
\left|O_{1}(t+d t)\right\rangle=\frac{-i \hat{H}^{(1)} d t|0(t)\rangle}{d p(t)}
$$

The time increment $d t$ should be chosen as the condition $d p(t) \ll 1$ being satisfied.

Averaging the processes $\left|0_{0}(t)\right\rangle$ and $\left|0_{1}(t)\right\rangle$ with respective probabilities 1 $d p(t)$ and $d p(t)$ :

$$
|0(t+d t)\rangle=[1-d p(t)]\left|0_{0}(t+d t)\right\rangle+d p(t)\left|0_{1}(t+d t)\right\rangle
$$

we can obtain the Fokker-Planck equation (7.8). Note that the ket-vacuums $\left|O_{0}(t)\right\rangle$ and $\left|O_{1}(t)\right\rangle$ look like satisfying a certain kind of stochastic Liouville equation.

\section{Summary}

The aim of this paper has been to study the system of QSDEs from a physical basis. We have formulated everything from the starting point using the method of NETFD. In the presented approach, boson and fermion systems are considered simultaneously. The obtained results have two fixed parameters: the real parameter $\sigma$ specifying different commutation rules for boson and fermion operators, and the complex parameter $\tau,(2.8)$, specifying different thermal state conditions for boson and fermion systems. Such a combined consideration was made possible due to the unification of fermion and boson stochastic calculus (Appendix B), where fermion annihilation and creation processes are realized in a Boson Fock space by means of a simple stochastic integral prescription leading to similar multiplication rules for stochastic differentials.

The dissipation mechanism is considered through the concept of a quantum noise, i.e. as a quantum field interacting with the relevant system. In our paper we considered two types of interaction with external fields: hermitian $(\lambda=1)$ and non-hermitian $(\lambda=0)$. With the latter, conservation of the probability is satisfied within the relevant system. With the former, information about only relevant system is not enough and instead of that we can speak about conservation of the probability within the total system: relevant system plus environment system. 
As we are concentrated on the stochastic equations, there are two types of stochastic calculus: Itô and Stratonovich. Correspondingly, equations used one or another type of stochastic calculus are classified as QSDE of the Itô or Stratonovich types. The Langevin equation of the Stratonovich type (6.65) has structure similar to one of the Heisenberg equation of motion for a dynamical quantity in quantum mechanics and quantum field theory. As a result of different stochastic multiplication rule, the Langevin equation of the Itô type (6.61) contains an extra term proportional to a product of random forces $d W_{t} d \tilde{W}_{t}$. The corresponding Fokker-Planck equation is then obtained most easily from the quantum stochastic Liouville equation of the Itô type by taking the random average. Though in fermion case the connection with the classical Brownian motion is only formal, the Itô/Stratonovich product formula is the same as in boson case (relations (A.15) and (A.16)). The averaged equation of motion for a dynamical quantity can be obtained in two ways. From the Langevin equation by taking both random average and the relevant vacuum expectation, or from the Fokker-Planck equation by taking the vacuum expectation of operators corresponding to the dynamical quantity. In our study we showed that QSDEs constructed upon hermitian and non-hermitian interaction hat-Hamiltonians lead to the same averaged equation of motion (6.84) for an arbitrary operator of the relevant system. In the case of stationary semi-free quantum stochastic process, its irreversibility is checked in terms of the Boltzmann entropy. We also demonstrated the relationship between the presented formulation and the method of quantum jump simulations.

The approach we followed in this article is rather formal and we are looking now for some demonstrative examples of its application for particular problems. An interesting result is obtained, for instance, for the model of a continuous quantum non-demolition measurement - continuous observation of a particle track in the cloud chamber $[95,96]$, and for the system corresponding to the quantum Kramers equation [97,98]. More detailed report about them will be presented elsewhere.

\section{Acknowledgments}

Authors would like to thank Mr. Y. Fukuda and Mr. Y. Kaburaki for their fruitful discussions.

\section{A Itô and Stratonovich calculus}

Definitions of the Itô [24] and Stratonovich [25] multiplications for arbitrary stochastic operators $X_{t}$ and $Y_{t}$ in the Schrödinger representation are given, 
respectively, by

$$
\begin{aligned}
& X_{t} \cdot d Y_{t}=X_{t}\left(Y_{t+d t}-Y_{t}\right) \\
& d X_{t} \cdot Y_{t}=\left(X_{t+d t}-X_{t}\right) Y_{t}
\end{aligned}
$$

and

$$
\begin{aligned}
X_{t} \circ d Y_{t} & =\frac{1}{2}\left(X_{t+d t}+X_{t}\right)\left(Y_{t+d t}-Y_{t}\right), \\
d X_{t} \circ Y_{t} & =\left(X_{t+d t}-X_{t}\right) \frac{1}{2}\left(Y_{t+d t}+Y_{t}\right) .
\end{aligned}
$$

From these relations we have the connection formulae between the Itô and Stratonovich products in the differential form as

$$
\begin{aligned}
& X_{t} \circ d Y_{t}=X_{t} \cdot d Y_{t}+\frac{1}{2} d X_{t} \cdot d Y_{t}, \\
& d X_{t} \circ Y_{t}=d X_{t} \cdot Y_{t}+\frac{1}{2} d X_{t} \cdot d Y_{t} .
\end{aligned}
$$

Note that random average of the stochastic multiplication (A.1) or (A.2) of the Itô type is equal to zero.

Definitions of the Itô and Stratonovich multiplications for stochastic operators $X(t)$ and $Y(t)$ in the Heisenberg representation are given in the same form by

$$
\begin{aligned}
& X(t) \cdot d Y(t)=X(t)[Y(t+d t)-Y(t)] \\
& d X(t) \cdot Y(t)=[X(t+d t)-X(t)] Y(t)
\end{aligned}
$$

and

$$
\begin{aligned}
& X(t) \circ d Y(t)=\frac{1}{2}[X(t+d t)+X(t)][Y(t+d t)-Y(t)] \\
& d X(t) \circ Y(t)=[X(t+d t)-X(t)] \frac{1}{2}[Y(t+d t)+Y(t)]
\end{aligned}
$$

where operators $X(t)$ and $d X(t)$ are introduced, respectively, through relations

$$
\begin{aligned}
X(t) & =\hat{V}_{F}^{-1}(t) X_{t} \hat{V}_{F}(t), \\
d X(t) & =d\left(\hat{V}_{F}^{-1}(t) X_{t} \hat{V}_{F}(t)\right),
\end{aligned}
$$

with $\hat{V}_{F}(t)$ being a stochastic time evolution operator. 
From (A.7) to (A.10), we have the connection formulae between the Itô and Stratonovich products in the differential form as

$$
\begin{aligned}
& X(t) \circ d Y(t)=X(t) \cdot d Y(t)+\frac{1}{2} d X(t) \cdot d Y(t) \\
& d X(t) \circ Y(t)=d X(t) \cdot Y(t)+\frac{1}{2} d X(t) \cdot d Y(t)
\end{aligned}
$$

Stochastic multiplications (A.7) to (A.10) are consistent with corresponding types of differential calculus for products of stochastic operators, which for the case of the Itô type calculus and the Stratonovich type calculus read, respectively, as

$$
d[X(t) Y(t)]=d X(t) \cdot Y(t)+X(t) \cdot d Y(t)+d X(t) \cdot d Y(t),
$$

and

$$
d[X(t) Y(t)]=d X(t) \circ Y(t)+X(t) \circ d Y(t)
$$

\section{B Boson and fermion Brownian motion}

Let $\Gamma_{\mathrm{s}}^{0}$ denotes the boson Fock space (the symmetric Fock space) over the Hilbert space $\mathcal{H}=L^{2}\left(\mathbf{R}_{+}\right)$of square integrable functions, and $b_{t}$ and $b_{t}^{\dagger}$ denote, respectively, boson annihilation and creation operators at time $t \in[0, \infty)$ satisfying the canonical commutation relations

$$
\left[b_{t}, b_{s}^{\dagger}\right]=\delta(t-s), \quad\left[b_{t}, b_{s}\right]=\left[b_{t}^{\dagger}, b_{s}^{\dagger}\right]=0 .
$$

The bra- and ket-vacuums (| and |) are defined, respectively, by

$$
\left(\left|b_{t}^{\dagger}=0, \quad b_{t}\right|\right)=0 .
$$

Note that $(|=|)^{\dagger}$ since here we are considering the unitary representation of $b_{t}$ and $b_{t}^{\dagger}$. The space $\Gamma_{\mathrm{s}}^{0}$ is equipped with a total family of exponential vectors

$$
\begin{aligned}
& \left(e(f) \mid=\left(\mid \exp \left\{\int_{0}^{\infty} d t f^{*}(t) b_{t}\right\},\right.\right. \\
& \left.\mid e(g))=\exp \left\{\int_{0}^{\infty} d t g(t) b_{t}^{\dagger}\right\} \mid\right),
\end{aligned}
$$


whose overlapping is

$$
(e(f) \mid e(g))=\exp \left\{\int_{0}^{\infty} d t f^{*}(t) g(t)\right\} .
$$

Here, $f, g \in \mathcal{H}$. The dense span of exponential vectors is denoted by $\mathcal{E}$. Operators $b_{t}, b_{t}^{\dagger}$ and exponential vectors are characterized by the relations

$$
\left(e(f)\left|b_{t}^{\dagger}=\left(e(f)\left|f^{*}(t), \quad b_{t}\right| e(g)\right)=g(t)\right| e(g)\right) .
$$

Let us introduce operator $U_{t}$ defined as

$$
U_{t}=\sigma_{<} P_{[0, t]}+\sigma_{>} P_{(t, \infty)},
$$

where $\sigma_{<}$and $\sigma_{>}$are two independent parameters taking values \pm 1 , and $P_{[a, b]}$ $(a \leq b)$ is an operator on $\mathcal{H}$ of multiplication by the indicator function whose action reads

$$
P_{[a, b]} \int_{0}^{\infty} d t g(t)=\int_{a}^{b} d t g(t)=\int_{0}^{\infty} d t \theta(t-a) \theta(b-t) g(t) .
$$

Here, $\theta(t)$ is the step function specified by

$$
\theta(t)=\left\{\begin{array}{l}
1 \text { for } t \geq 0 \\
0 \text { for } t<0
\end{array}\right.
$$

The operator $P_{[a, b]}$ has the following properties:

$$
P_{[a, b]}^{2}=P_{[a, b]}, \quad P_{[a, b]}^{\dagger}=P_{[a, b]}, \quad P_{[a, b]} P_{[c, d]}=P_{[c, d]} P_{[a, b]},
$$

which are easily verified using the definition (B.8). Then, we see that operator $U_{t}$ is unitary, and satisfies

$$
U_{t}^{2}=I, \quad U_{t}^{\dagger}=U_{t}, \quad U_{t} U_{s}=U_{s} U_{t},
$$

where $I$ is the identity operator.

The so-called reflection process $J_{t} \equiv J_{t}\left(U_{t}\right), t \in \mathbf{R}_{+}$, whose action on $\mathcal{E}$ is given by [62] 


$$
\left.\left.\left.J_{t} \mid e(g)\right)=\mid e\left(U_{t} g\right)\right)=\exp \left\{U_{t} \int_{0}^{\infty} d t^{\prime} g\left(t^{\prime}\right) b_{t^{\prime}}^{\dagger}\right\} \mid\right)
$$

inherits properties of the operator $U_{t}$, (B.11), i.e.

$$
J_{t}^{2}=1, \quad J_{t}^{\dagger}=J_{t}, \quad J_{t} J_{s}=J_{s} J_{t}
$$

and does not change the vacuum:

$$
\left(\left|J_{t}=\left(\left|, \quad J_{t}\right|\right)=\right|\right) .
$$

Here, $\mathbf{1}$ is the unit operator defined in $\Gamma_{\mathrm{s}}^{0}$.

Let us now consider new operators

$$
\mathrm{b}_{t}=J_{t} b_{t}, \quad \mathrm{~b}_{t}^{\dagger}=b_{t}^{\dagger} J_{t} .
$$

Apparently, they annihilate vacuums

$$
\left(\left|\mathbf{b}_{t}^{\dagger}=0, \quad \mathrm{~b}_{t}\right|\right)=0
$$

The following matrix elements

$$
\begin{aligned}
\left(e(f)\left|\left[J_{t}, b_{s}\right]_{-\sigma}\right| e(g)\right)= & (e(f) \mid\{1 \\
& \left.\left.-\sigma\left[\sigma_{>}+\left(\sigma_{<}-\sigma_{>}\right) \theta(t-s)\right]\right\} g(s) J_{t} \mid e(g)\right), \\
\left(e(f)\left|\left[\mathbf{b}_{t}, \mathbf{b}_{s}^{\dagger}\right]_{-\sigma}\right| e(g)\right)= & (e(f)|\delta(t-s)| e(g)) \\
& +\left(e(f)\left|J_{t} J_{s}\left(\sigma_{>} \sigma_{<}-\sigma\right) f^{*}(s) g(t)\right| e(g)\right),
\end{aligned}
$$

are valid for $f, g \in \mathcal{H}$. Then the requirement of equal-time (anti-)commutativity between $J_{t}$ and $b_{t}$

$$
\left[J_{t}, b_{t}\right]_{-\sigma}=0
$$

gives

$$
1-\sigma \sigma_{<}=0
$$

while the requirement of canonical (anti-)commutation relation

$$
\left[\mathrm{b}_{t}, \mathrm{~b}_{s}^{\dagger}\right]_{-\sigma}=\delta(t-s)
$$


leads to

$$
\sigma_{>} \sigma_{<}-\sigma=0
$$

All those conditions are satisfied when $\sigma_{<}=\sigma$ and $\sigma_{>}=+1$. Then the operator $U_{t}$ turns out to be

$$
U_{t}=\sigma P_{[0, t]}+P_{(t, \infty)}
$$

Note that for a boson system, i.e. $\sigma=1, U_{t}=I$ and the operators $\mathrm{b}_{t}$ and $\mathrm{b}_{t}^{\dagger}$ reduce, respectively, to $b_{t}$ and $b_{t}^{\dagger}$.

We see that the generalized quantum Brownian motion, defined by

$$
\mathrm{B}_{t}=\int_{0}^{t} d t^{\prime} \mathrm{b}_{t^{\prime}}, \quad \mathrm{B}_{t}^{\dagger}=\int_{0}^{t} d t^{\prime} \mathrm{b}_{t^{\prime}}^{\dagger}
$$

with $\mathrm{B}_{0}=0, \mathrm{~B}_{0}^{\dagger}=0$, satisfies

$$
\left[\mathrm{B}_{t}, \mathrm{~B}_{s}^{\dagger}\right]_{-\sigma}=\min (t, s)
$$

The case $\sigma=1$ represents the boson Brownian motion [49,55], whereas the case $\sigma=-1$ the fermion Brownian motion [62]. Their increments

$$
\begin{aligned}
& d \mathrm{~B}_{t}=\mathrm{B}_{t+d t}-\mathrm{B}_{t}=\mathrm{b}_{t} d t \\
& d \mathrm{~B}_{t}^{\dagger}=\mathrm{B}_{t+d t}^{\dagger}-\mathrm{B}_{t}^{\dagger}=\mathrm{b}_{t}^{\dagger} d t
\end{aligned}
$$

annihilate the vacuum, i.e.

$$
\left(\left|d \mathrm{~B}_{t}^{\dagger}=0, \quad d \mathrm{~B}_{t}\right|\right)=0,
$$

and their matrix elements read

$$
\begin{aligned}
\left(e(f)\left|d \mathrm{~B}_{t}\right| e(g)\right) & =\left(e(f)\left|J_{t} g(t) d t\right| e(g)\right) \\
\left(e(f)\left|d \mathrm{~B}_{t}^{\dagger}\right| e(g)\right) & =\left(e(f)\left|f^{*}(t) d t J_{t}\right| e(g)\right) \\
\left(e(f)\left|d \mathrm{~B}_{t} d \mathrm{~B}_{t}\right| e(g)\right) & =0 \\
\left(e(f)\left|d \mathrm{~B}_{t}^{\dagger} d \mathrm{~B}_{t}\right| e(g)\right) & =0 \\
\left(e(f)\left|d \mathrm{~B}_{t} d \mathrm{~B}_{t}^{\dagger}\right| e(g)\right) & =d t(e(f) \mid e(g))
\end{aligned}
$$


Here we neglected terms of the higher order than $d t$. The latter equations are summarized in the following table of multiplication rules for increments $d \mathrm{~B}_{t}$ and $d \mathrm{~B}_{t}^{\dagger}$ :

\begin{tabular}{c|lcc} 
& $d \mathrm{~B}_{t}$ & $d \mathrm{~B}_{t}^{\dagger}$ & $d t$ \\
\hline$d \mathrm{~B}_{t}$ & 0 & $d t$ & 0 \\
$d \mathrm{~B}_{t}^{\dagger}$ & 0 & 0 & 0 \\
$d t$ & 0 & 0 & 0
\end{tabular}

Now we consider a tensor product space $\hat{\Gamma}=\Gamma_{s}^{0} \otimes \tilde{\Gamma}_{s}^{0}$. Its vacuum states $\left.\left.\mid\right)\right)$ and exponential vectors $\mid e(f, g)))$ are defined through the "principle of correspondence" [3]

$$
\begin{aligned}
\mid)) & \longleftrightarrow \mid)(\mid, \\
\mid e(f, g))) & \longleftrightarrow \mid e(f))(e(g) \mid .
\end{aligned}
$$

Annihilation and creation operators acting on $\hat{\Gamma}$ are defined through

$$
\begin{aligned}
\left.\left.b_{t} \mid e(f, g)\right)\right) & \left.\longleftrightarrow b_{t} \mid e(f)\right)(e(g) \mid, \\
\left.\left.b_{t}^{\dagger} \mid e(f, g)\right)\right) & \left.\longleftrightarrow b_{t}^{\dagger} \mid e(f)\right)(e(g) \mid, \\
\left.\left.\tilde{b}_{t} \mid e(f, g)\right)\right) & \longleftrightarrow \mid e(f))\left(e(g) \mid b_{t}^{\dagger},\right. \\
\left.\left.\tilde{b}_{t}^{\dagger} \mid e(f, g)\right)\right) & \longleftrightarrow(e(f))\left(e(g) \mid b_{t},\right.
\end{aligned}
$$

and similarly for $J_{t}$ and $\tilde{J}_{t}$, i.e.

$$
\begin{aligned}
& \left.\left.\left.J_{t} \mid e(f, g)\right)\right) \longleftrightarrow J_{t} \mid e(f)\right)(e(g) \mid \\
& \left.\left.\left.\tilde{J}_{t} \mid e(f, g)\right)\right) \longleftrightarrow \mid e(f)\right)\left(e(g) \mid J_{t} .\right.
\end{aligned}
$$

Algebra of commutation relations between these operators reads

$$
\begin{aligned}
{\left[b_{t}, b_{s}^{\dagger}\right] } & =\left[\tilde{b}_{t}, \tilde{b}_{s}^{\dagger}\right]=\delta(t-s), \\
{\left[b_{t}, \tilde{b}_{s}\right] } & =\left[b_{t}, \tilde{b}_{s}^{\dagger}\right]=0 \\
{\left[J_{t}, \tilde{b}_{s}\right] } & =\left[\tilde{J}_{t}, b_{s}\right]=0 \\
{\left[J_{t}, b_{t}\right]_{-\sigma} } & =\left[\tilde{J}_{t}, \tilde{b}_{t}\right]_{-\sigma}=0 .
\end{aligned}
$$

Let us now consider new operators defined by 


$$
\begin{aligned}
& \mathrm{b}_{t}=J_{t} b_{t}, \quad \mathrm{~b}_{t}^{\dagger}=b_{t}^{\dagger} J_{t}, \\
& \tilde{\mathrm{b}}_{t}=\hat{\tau} \tilde{J}_{t} \tilde{b}_{t}, \quad \tilde{\mathrm{b}}_{t}^{\dagger}=\hat{\tau} \tilde{b}_{t}^{\dagger} \tilde{J}_{t},
\end{aligned}
$$

where $\hat{\tau}$ is an operator satisfying the following (anti-)commutation relations

$$
\begin{aligned}
{\left[\hat{\tau}, J_{t}\right] } & =\left[\hat{\tau}, \tilde{J}_{t}\right]=0, \\
{\left[\hat{\tau}, b_{t}\right]_{-\sigma} } & =\left[\hat{\tau}, b_{t}^{\dagger}\right]_{-\sigma}=0, \\
{\left[\hat{\tau}, \tilde{b}_{t}\right]_{-\sigma} } & =\left[\hat{\tau}, \tilde{b}_{t}^{\dagger}\right]_{-\sigma}=0,
\end{aligned}
$$

and the condition

$$
\hat{\tau}^{2}=\sigma
$$

Operators $\mathrm{b}_{t}, \mathrm{~b}_{t}^{\dagger}$ and their tilde conjugates annihilate vacuums

$$
\left(\left(\left|\mathrm{b}_{t}^{\dagger}=\left(\left(\left|\tilde{\mathrm{b}}_{t}^{\dagger}=0, \quad \mathrm{~b}_{t}\right|\right)\right)=\tilde{\mathrm{b}}_{t}\right|\right)\right)=0
$$

and satisfy canonical (anti-)commutation relations

$$
\begin{aligned}
& {\left[\mathrm{b}_{t}, \mathrm{~b}_{s}^{\dagger}\right]_{-\sigma}=\left[\tilde{\mathrm{b}}_{t}, \tilde{\mathrm{b}}_{s}^{\dagger}\right]_{-\sigma}=\delta(t-s),} \\
& {\left[\mathrm{b}_{t}, \tilde{\mathrm{b}}_{s}\right]_{-\sigma}=\left[\mathrm{b}_{t}, \tilde{\mathrm{b}}_{s}^{\dagger}\right]_{-\sigma}=0 .}
\end{aligned}
$$

Since $\left(\tilde{\mathbf{b}}_{t}\right)^{\dagger}$ and $\left(\mathbf{b}_{t}^{\dagger}\right)^{\sim}$ are calculated as

$$
\begin{aligned}
\left(\tilde{\mathrm{b}}_{t}\right)^{\dagger} & =\tilde{b}_{t}^{\dagger} \tilde{J}_{t} \hat{\tau}^{\dagger}=\sigma \hat{\tau}^{\dagger} \tilde{b}_{t}^{\dagger} \tilde{J}_{t}, \\
\left(\mathrm{~b}_{t}^{\dagger}\right)^{\sim} & =\hat{\tau}\left(b_{t}^{\dagger} J_{t}\right)^{\sim}=\hat{\tau} \tilde{b}_{t}^{\dagger} \tilde{J}_{t},
\end{aligned}
$$

the commutativity of tilde conjugation and hermitian conjugation for operators (B.47) and (B.48) implies

$$
\hat{\tau}^{\dagger}=\sigma \hat{\tau}
$$

In order to fulfill the requirement that double tilde conjugation applied to operators $\mathrm{b}_{t}$ 's leaves them unchanged one needs to put

$$
(\hat{\tau})^{\sim}=\hat{\tau}^{\dagger}
$$

since

$$
\left(\tilde{b}_{t}\right)^{\sim}=\hat{\tau}(\hat{\tau})^{\sim} J_{t} b_{t}=\hat{\tau}(\hat{\tau})^{\sim} b_{t}
$$


Because of

$$
\left.\left.\left.\tilde{\mathrm{b}}_{t}()\right)=\tilde{b}_{t}(\hat{\tau} \mid)\right)\right)=0
$$

one can conclude that $\hat{\tau} \mid)) \propto(\mid))$. The proportionality factor is a phase factor since the norm of $\hat{\tau} \mid)$ ) is unity:

$$
\left(\left(\left|\hat{\tau}^{\dagger} \hat{\tau}\right|\right)\right)=((|\sigma \hat{\tau} \hat{\tau}|))=\sigma^{2}=1 .
$$

Hence one can write

$$
\left.\left.\hat{\tau} \mid))=\mathrm{e}^{i \phi / 2} \mid\right)\right) .
$$

Multiplying both sides by $\hat{\tau}$, one has

$$
\left.\left.\left.\left.\left.\hat{\tau}^{2} \mid\right)\right)=\mathrm{e}^{i \phi}(\mid)\right)=\sigma \mid\right)\right)
$$

which gives $\mathrm{e}^{i \phi}=\sigma$, or

$$
\hat{\tau} \mid))=\sqrt{\sigma}()) .
$$

Thermal degree of freedom can be introduced by the Bogoliubov transformation in $\hat{\Gamma}$. For this purpose we require that the expectation value of $b_{t}^{\dagger} b_{s}$ should be

$$
\left\langle\mathrm{b}_{t}^{\dagger} \mathrm{b}_{s}\right\rangle=\bar{n} \delta(t-s)
$$

with $\bar{n} \in \mathbf{R}_{+}$, where $\langle\cdots\rangle=\langle|\cdots|\rangle$ indicates the expectation with respect to tilde invariant thermal vacuums \langle| and |\rangle . The requirement (B.66) is consistent with TSC for states \langle| and |\rangle such that

$$
\left\langle\left|\tilde{\mathbf{b}}_{t}^{\dagger}=\tau^{*}\left\langle\left|\mathbf{b}_{t}, \quad \tilde{\mathbf{b}}_{t}\right|\right\rangle=\frac{\tau \bar{n}}{1+\sigma \bar{n}} \mathbf{b}_{t}^{\dagger}\right|\right\rangle
$$

Let us introduce annihilation and creation operators

$$
\begin{aligned}
\mathrm{c}_{t} & =[1+\sigma \bar{n}] \mathrm{b}_{t}-\sigma \tau \bar{n} \tilde{\mathrm{b}}_{t}^{\dagger}, \\
\tilde{\mathrm{c}}_{t}^{\text {q }} & =\tilde{\mathrm{b}}_{t}^{\dagger}-\sigma \tau \mathrm{b}_{t},
\end{aligned}
$$

and their tilde conjugates. From the TSC (B.67) one has 


$$
\left\langle\left|c_{t}^{+}=\left\langle\left|\tilde{c}_{t}^{+}=0, \quad c_{t}\right|\right\rangle=\tilde{c}_{t}\right|\right\rangle=0 .
$$

With the thermal doublet notations

$$
\overline{\mathrm{b}}_{t}^{\mu}=\left(\mathrm{b}_{t}^{\dagger},-\tau \tilde{\mathrm{b}}_{t}\right), \quad \mathrm{b}_{t}^{\nu}=\text { collon }\left(\mathrm{b}_{t}, \tau \tilde{\mathrm{b}}_{t}^{\dagger}\right)
$$

and

$$
\overline{\mathrm{c}}_{t}^{\mu}=\left(\mathrm{c}_{t}^{\text {p }},-\tau \tilde{\mathrm{c}}_{t}\right), \quad \mathrm{c}_{t}^{\nu}=\text { collon }\left(\mathrm{c}_{t}, \tau \tilde{\mathrm{c}}_{t}^{\text {p}}\right),
$$

(B.68), (B.69) and their tilde conjugates can be written in form of the Bogoliubov transformation

$$
\mathrm{c}_{t}^{\mu}=B^{\mu \nu} \mathrm{b}_{t}^{\nu}, \quad \overline{\mathrm{c}}_{t}^{\nu}=\overline{\mathrm{b}}_{t}^{\mu}\left[B^{-1}\right]^{\mu \nu},
$$

with (7.12). This new operators satisfy the canonical (anti-)commutation relations

$$
\left[\mathrm{c}_{t}, \mathrm{c}_{s}^{+}\right]_{-\sigma}=\delta(t-s)
$$

In the following, we will use the representation space constructed on vacuums \langle| and |\rangle . Note that $\langle|\neq|\rangle^{\dagger}$, i.e. it is not a unitary representation. Let $\hat{\Gamma}^{\beta}$ denotes the Fock space spanned by the basic bra- and ket-vectors introduced by a cyclic operations of $c_{t}, \tilde{c}_{t}$ on the thermal bra-vacuum \langle| , and of $c_{t}^{+}$, $\tilde{c}_{t}^{\text {q }}$ on the thermal ket-vacuum |\rangle . Quantum Brownian motion at finite temperature is defined in the Fock space $\hat{\Gamma}^{\beta}$ by operators

$$
\mathrm{B}_{t}^{\sharp}=\int_{0}^{t} d s \mathbf{b}_{s}^{\sharp}, \quad \tilde{\mathrm{B}}_{t}^{\sharp}=\int_{0}^{t} d s \tilde{\mathbf{b}}_{s}^{\sharp},
$$

with $B_{0}^{\sharp}=0$ and $\tilde{B}_{0}^{\sharp}=0$, where $\sharp$ stands for null or dagger. The explicit representation of processes $B_{t}^{\sharp}$ and $\tilde{B}_{t}^{\sharp}$ can be performed in terms of the Bogoliubov transformation. The couple $\mathrm{B}_{t}$ and $\mathrm{B}_{t}^{\dagger}$, for example, is calculated as

$$
\begin{aligned}
& \mathrm{B}_{t}=\int_{0}^{t} d s\left(\mathrm{c}_{s}+\sigma \tau \bar{n} \tilde{\mathrm{c}}_{s}^{+}\right)=\mathrm{C}_{t}+\sigma \tau \bar{n} \tilde{\mathrm{C}}_{t}^{+}, \\
& \mathrm{B}_{t}^{\dagger}=\int_{0}^{t} d s\left([1+\sigma \bar{n}] \mathrm{c}_{s}^{+}+\tau \tilde{\mathrm{C}}_{s}\right)=[1+\sigma \bar{n}] \mathrm{C}_{t}^{+}+\tau \tilde{\mathrm{C}}_{t},
\end{aligned}
$$

where we defined new operators 


$$
\mathrm{C}_{t}^{\sharp}=\int_{0}^{t} d s \mathrm{C}_{s}^{\sharp}, \quad \tilde{\mathrm{C}}_{t}^{\sharp}=\int_{0}^{t} d s \tilde{\mathrm{C}}_{s}^{\sharp},
$$

with $C_{0}^{\sharp}=0$ and $\tilde{C}_{0}^{\sharp}=0$, and $\sharp$ standing for null or the Venus-mark. Since matrix elements of $d C_{t}^{\sharp}$ and $d \tilde{C}_{t}^{\sharp}$ in thermal space $\hat{\Gamma}^{\beta}$ read

$$
\begin{aligned}
\left\langle d \mathrm{C}_{t}\right\rangle=\left\langle d \tilde{\mathrm{C}}_{t}\right\rangle & =\left\langle d \mathrm{C}_{t}^{+}\right\rangle=\left\langle d \tilde{\mathrm{C}}_{t}^{+}\right\rangle=0, \\
\left\langle d \mathrm{C}_{t}^{+} d \mathrm{C}_{t}\right\rangle & =\left\langle d \tilde{\mathrm{C}}_{t}^{+} d \tilde{\mathrm{C}}_{t}\right\rangle=0, \\
\left\langle d \mathrm{C}_{t} d \mathrm{C}_{t}^{+}\right\rangle & =\left\langle d \tilde{\mathrm{C}}_{t} d \tilde{\mathrm{C}}_{t}^{+}\right\rangle=d t,
\end{aligned}
$$

calculation of moments of quantum Brownian motion in the thermal space $\hat{\Gamma}^{\beta}$ can be performed, for instance, as

$$
\begin{aligned}
\left\langle d \mathrm{~B}_{t} d \mathrm{~B}_{t}^{\dagger}\right\rangle & =\left\langle\left(d \mathrm{C}_{t}+\sigma \tau \bar{n} d \tilde{\mathrm{C}}_{t}^{+}\right)\left([1+\sigma \bar{n}] d \mathrm{C}_{t}^{+}+\tau d \tilde{\mathrm{C}}_{t}\right)\right\rangle \\
& =[1+\sigma \bar{n}]\left\langle d \mathrm{C}_{t} d \mathrm{C}_{t}^{+}\right\rangle=[1+\sigma \bar{n}] d t .
\end{aligned}
$$

Repeating this for other pair products of $d \mathrm{~B}_{t}^{\sharp}, d \tilde{\mathrm{B}}_{t}^{\sharp}$ and $d t$, multiplication rules for these increments can be summarized in the following table:

\begin{tabular}{c|ccccc} 
& $d \mathrm{~B}_{t}$ & $d \mathrm{~B}_{t}^{\dagger}$ & $d \tilde{\mathrm{B}}_{t}$ & $d \tilde{\mathrm{B}}_{t}^{\dagger}$ & $d t$ \\
\hline$d \mathrm{~B}_{t}$ & 0 & {$[1+\sigma \bar{n}] d t$} & $\tau \bar{n} d t$ & 0 & 0 \\
$d \mathrm{~B}_{t}^{\dagger}$ & $\bar{n} d t$ & 0 & 0 & $\tau[1+\sigma \bar{n}] d t$ & 0 \\
$d \tilde{\mathrm{B}}_{t}$ & $\sigma \tau \bar{n} d t$ & 0 & 0 & {$[1+\sigma \bar{n}] d t$} & 0 \\
$d \tilde{\mathrm{B}}_{t}^{\dagger}$ & 0 & $\sigma \tau[1+\sigma \bar{n}] d t$ & $\bar{n} d t$ & 0 & 0 \\
$d t$ & 0 & 0 & 0 & 0 & 0
\end{tabular}

\section{Treatment of fermions in Thermo Field Dynamics}

We are deciding the double tilde conjugation rule and the thermal state conditions for fermions $[99,100]$ by considering the system consisting of a vector field and Faddeev-Popov ghosts [101].

In the case of pure Abelian gauge field within the Feynman gauge, the system is specified by the Hamiltonian $H_{\mathrm{vf}+\mathrm{gh}}=H_{\mathrm{vf}}+H_{\mathrm{gh}}$ defined on the total state vector space $\mathcal{V}=\mathcal{V}_{\mathrm{vf}} \otimes \mathcal{V}_{\mathrm{gh}} . H_{\mathrm{vf}}$ and $H_{\mathrm{gh}}$ are, respectively, Hamiltonians for the vector field and ghosts defined on the vector field sector $\mathcal{V}_{\mathrm{vf}}$ and the ghost sector $\mathcal{V}_{\text {gh }}$ given by 


$$
H_{\mathrm{vf}}=-\int d^{3} k \varepsilon(\vec{k}) g^{\mu \nu} a_{\mu}^{\dagger}(\vec{k}) a_{\nu}(\vec{k}),
$$

with $\varepsilon(\vec{k})=|\vec{k}|$ being the energy spectrum and $g^{\mu \nu}=\operatorname{diag}(1,-1,-1,-1)$, and by

$$
H_{\mathrm{gh}}=-i \int d^{3} k \varepsilon(\vec{k})\left[\bar{c}^{\dagger}(\vec{k}) c(\vec{k})-c^{\dagger}(\vec{k}) \bar{c}(\vec{k})\right] .
$$

Here, $a_{\mu}^{\dagger}(\vec{k})$ and $a_{\mu}(\vec{k})$ are, respectively, creation and annihilation operators of the gauge field of the mode $\vec{k}$ satisfying the canonical commutation relations

$$
\left[a_{\mu}(\vec{k}), a_{\nu}^{\dagger}(\vec{q})\right]=-g_{\mu \nu} \delta^{3}(\vec{k}-\vec{q})
$$

while $c^{\dagger}(\vec{k})$ and $c(\vec{k})\left[\bar{c}^{\dagger}(\vec{k})\right.$ and $\left.\bar{c}(\vec{k})\right]$ are, respectively, creation and annihilation operators of ghosts [anti-ghosts] satisfying the following canonical anticommutation relations:

$$
\left[c(\vec{k}), \bar{c}^{\dagger}(\vec{q})\right]_{+}=-\left[\bar{c}(\vec{k}), c^{\dagger}(\vec{q})\right]_{+}=i \delta^{3}(\vec{k}-\vec{q}) .
$$

Other combinations of ghost/anti-ghost operators anti-commute with each other. The BRS charge - generator of the BRS transformation [102], and the ghost charge $[103,104]$ acting on the total space $\mathcal{V}$ are, respectively, given by

$$
\begin{aligned}
Q_{B} & =-\int d^{3} k k^{\mu}\left[a_{\mu}(\vec{k}) c^{\dagger}(\vec{k})+a_{\mu}^{\dagger}(\vec{k}) c(\vec{k})\right], \\
Q_{c} & =\int d^{3} k\left[c^{\dagger}(\vec{k}) \bar{c}(\vec{k})+\bar{c}^{\dagger}(\vec{k}) c(\vec{k})\right]
\end{aligned}
$$

which satisfy

$$
\left[i Q_{c}, Q_{B}\right]=Q_{B}
$$

Let us introduce a set of new operators $\left\{a_{(\sigma)}(\vec{k}) \mid \sigma=+,-, \mathrm{L}, \mathrm{S}\right\}$ through the relation

$$
a_{\mu}(\vec{k})=a_{(\sigma)}(\vec{k}) \epsilon_{\mu}^{(\sigma)}(\vec{k})
$$

where $\epsilon_{\mu}^{(\sigma)}(\vec{k})$ are polarization vectors defined by

$$
\epsilon_{\mu}^{( \pm)}(\vec{k})=\left(0, \vec{e}_{ \pm}\right)
$$




$$
\begin{aligned}
\epsilon_{\mu}^{(\mathrm{L})}(\vec{k}) & =-i k_{\mu}=-i(|\vec{k}|, \vec{k}), \\
\epsilon_{\mu}^{(\mathrm{S})}(\vec{k}) & =-i \bar{k}_{\mu} / 2|\vec{k}|^{2}=-i(|\vec{k}|,-\vec{k}) / 2|\vec{k}|^{2}
\end{aligned}
$$

with $\vec{e}_{ \pm}$satisfying $\vec{e}_{ \pm} \cdot \vec{k}=0, \vec{e}_{ \pm}^{*} \cdot \vec{e}_{\mp}=0$ and $\vec{e}_{ \pm}^{*} \cdot \vec{e}_{ \pm}=1$. The polarization vectors $\epsilon_{\mu}^{( \pm)}(\vec{k})$ correspond, respectively, to the transverse modes with helicity \pm 1 , while $\epsilon_{\mu}^{(\mathrm{L})}(\vec{k})$ and $\epsilon_{\mu}^{(\mathrm{S})}(\vec{k})$ indicate, respectively, the longitudinal mode and the scalar mode. With the definition (C.10) and (C.11), we see that $\epsilon_{\mu}^{(\mathrm{L}) *(\vec{k})}$.

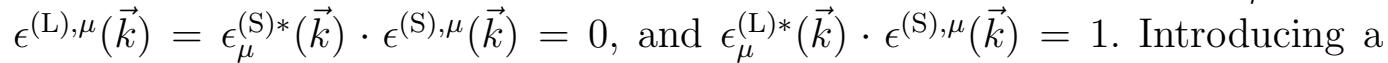
"metric"

$$
g^{(\sigma \tau)}=g_{(\sigma \tau)}=\left(\begin{array}{rrrr}
-1 & 0 & 0 & 0 \\
0 & -1 & 0 & 0 \\
0 & 0 & 0 & 1 \\
0 & 0 & 1 & 0
\end{array}\right)
$$

we can define "contravariant" polarization vectors through $\epsilon_{(\sigma)}^{\mu}(\vec{k})=g_{(\sigma \tau)} \epsilon^{(\tau), \mu}(\vec{k})$, and see that

$$
\begin{gathered}
\epsilon_{(\sigma)}^{\mu *}(\vec{k}) \cdot \epsilon^{(\sigma), \nu}(\vec{k})=g^{\mu \nu}, \\
\epsilon_{\mu}^{(\sigma) *}(\vec{k}) \cdot \epsilon^{(\tau), \mu}(\vec{k})=g^{(\sigma \tau)} .
\end{gathered}
$$

The commutation relations (C.3) being rewritten in terms of operators $a_{(\sigma)}(\vec{k})$ and $a_{(\sigma)}^{\dagger}(\vec{k})$ become

$$
\left[a_{(\sigma)}(\vec{k}), a_{(\tau)}^{\dagger}(\vec{q})\right]=-g_{(\sigma \tau)} \delta(\vec{k}-\vec{q}) .
$$

Also the Hamiltonian for the vector field and generator of the BRS transformation read

$$
\begin{aligned}
& H_{\mathrm{vf}}=-\int d^{3} k \varepsilon(\vec{k}) g^{(\sigma \tau)} a_{(\sigma)}^{\dagger}(\vec{k}) a_{(\tau)}(\vec{k}), \\
& Q_{B}=-i \int d^{3} k\left[a_{(\mathrm{S})}^{\dagger}(\vec{k}) c(\vec{k})-a_{(\mathrm{S})}(\vec{k}) c^{\dagger}(\vec{k})\right] .
\end{aligned}
$$

In the local covariant operator formalism $[103,104]$ of gauge theories, the space $\mathcal{V}$ of state vectors has inevitably an indefinite metric as can be seen by (C.15) with (C.12). The physical subspace $\mathcal{V}_{\text {phys }}$ of $\mathcal{V}$, defined by [104]

$$
Q_{B} \mathcal{V}_{\text {phys }}=0
$$


can be shown to have a positive semi-definite metric [104]. Dividing $\mathcal{V}_{\text {phys }}$ by its subspace $\mathcal{V}_{0}$ consisting of normless states, we have, as a quotient space, the physical Hilbert space $H_{\text {phys }}\left(=\mathcal{V}_{\text {phys }} / \mathcal{V}_{0}\right)$ with positive definite metric in which the probabilistic interpretation of quantum theory works. $H_{\text {phys }}$ is isomorphic to the Hilbert space $\mathcal{H}_{\text {phys }}$ spanned by the Fock states created by the cyclic operation of $a_{( \pm)}^{\dagger}(\vec{k})$ on a certain vacuum. The space spanned by the Fock states created by $a_{(\mathrm{S})}^{\dagger}(\vec{k})$ is classified in $\mathcal{V}_{0}$, while the space spanned by the Fock states created by $a_{(\mathrm{L})}^{\dagger}(\vec{k})$ is classified in a space complemented to $\mathcal{V}_{\text {phys }}$. This reflects the fact that physical modes for photons are two transverse modes only.

As it is sufficient to pay attention to one mode in the following manipulation, we will pick up a mode, say $\vec{k}$, from each type of particles, and drop the index $\vec{k}$, for simplicity. Let us span the state vector space $\mathcal{V}$ by means of a set of the bases $\left.\left.\left\{\mid\left\{n_{(\sigma)}\right\}\right) \cdot \mid n_{c}, n_{\bar{c}}\right)\right\}$ whose elements, being the bases of the vector field sector $\mathcal{V}_{\mathrm{vf}}$ and the ghost sector $\mathcal{V}_{\text {gh }}$, respectively, are defined by

$$
\begin{aligned}
\left.\mid\left\{n_{(\sigma)}\right\}\right) & \left.=\prod_{\sigma= \pm, \mathrm{L}, \mathrm{S}} \frac{1}{\sqrt{n_{(\sigma)} !}}\left(a_{(\sigma)}^{\dagger}\right)^{n_{(\sigma)}} \mid\{0\}\right), \\
\left.\mid n_{c}, n_{\bar{c}}\right) & \left.=\left(c^{\dagger}\right)^{n_{c}}\left(\bar{c}^{\dagger}\right)^{n_{\bar{c}}} \mid 0,0\right) .
\end{aligned}
$$

They constitute the eigenstates of $H_{\mathrm{vf}}, H_{\mathrm{gh}}$ and $i Q_{c}$ :

$$
\begin{aligned}
\left.H_{\mathrm{vf}} \mid\left\{n_{(\sigma)}\right\}\right) & \left.=E_{\mathrm{vf}}\left(\left\{n_{(\sigma)}\right\}\right) \mid\left\{n_{(\sigma)}\right\}\right), \\
\left.H_{\mathrm{gh}} \mid n_{c}, n_{\bar{c}}\right) & \left.=E_{\mathrm{gh}}\left(n_{c}, n_{\bar{c}}\right) \mid n_{c}, n_{\bar{c}}\right), \\
\left.i Q_{c} \mid n_{c}, n_{\bar{c}}\right) & \left.=N_{\mathrm{gh}}\left(n_{c}, n_{\bar{c}}\right) \mid n_{c}, n_{\bar{c}}\right),
\end{aligned}
$$

with $E_{\mathrm{vf}}\left(\left\{n_{(\sigma)}\right\}\right)=\varepsilon \sum_{\sigma} n_{(\sigma)}, E_{\mathrm{gh}}\left(n_{c}, n_{\bar{c}}\right)=\varepsilon\left(n_{c}+n_{\bar{c}}\right)$ and $N_{\mathrm{gh}}\left(n_{c}, n_{\bar{c}}\right)=$ $\left(n_{c}-n_{\bar{c}}\right)$, where $n_{(\sigma)}$ are non-negative integers, and $n_{c}$ and $n_{\bar{c}}$ take values of 0 or 1 . We will denote the basis vectors $\left.\left.\left\{\mid\left\{n_{(\sigma)}\right\}\right) \cdot \mid n_{c}, n_{\bar{c}}\right)\right\}$ by $\left.\{\mid n)\right\}$ for brevity. Then, the metric tensor of $\mathcal{V}$ is

$$
\eta_{n, m}=(n \mid m)
$$

At the finite temperature, the statistical average of an observable quantity $A$, satisfying

$$
\left[Q_{B}, A\right]=0
$$

is given by [105] 


$$
\langle A\rangle=\operatorname{Tr} A \rho P^{(0)}=\operatorname{Tr} A \rho \mathrm{e}^{\pi Q_{c}}
$$

with $P^{(0)}$ being a projection operator onto $\mathcal{H}_{\text {phys }}$ and $\rho=Z^{-1} \mathrm{e}^{-\beta H_{\mathrm{vf}+\mathrm{gh}}}$ being the statistical operator acting on $\mathcal{V}$ with the partition function $Z=$ $\operatorname{Tr} \mathrm{e}^{-\beta H_{\mathrm{vf}+\mathrm{gh}}+\pi Q_{c}}$; the trace operation is taken in the space $\mathcal{V}$. Here, for the second equality in (C.26), we used the BRS-invariance of the statistical operator

$$
\left[Q_{B}, \rho\right]=0
$$

Let us express the statistical average (C.26) as the vacuum expectation in the doubled state space (thermal space) $\hat{\mathcal{V}}=\mathcal{V} \otimes \tilde{\mathcal{V}}$ which is introduced as follows. If $A$ is an operator on $\mathcal{V}$ so that

$$
\left.A=\sum_{n, m} A_{n m} \mid n\right)(m \mid
$$

the corresponding vector $|A\rangle$ in $\hat{\mathcal{V}}$ is obtained as

$$
|A\rangle=A_{n m}|n, \tilde{m}\rangle
$$

where $\left\{|n, \tilde{m}\rangle \equiv\left|\left\{n_{(\sigma)}\right\},\left\{\tilde{m}_{(\sigma)}\right\}\right\rangle \cdot\left|n_{c}, n_{\bar{c}}, \tilde{m}_{c}, \tilde{m}_{\bar{c}}\right\rangle\right\}$ is the set of the bases spanning $\hat{\mathcal{V}}$ and defined through the "principle of correspondence" [3]:

$$
\begin{aligned}
&\left|\left\{n_{(\sigma)}\right\},\left\{\tilde{m}_{(\sigma)}\right\}\right\rangle\left.\longleftrightarrow \mid\left\{n_{(\sigma)}\right\}\right)\left(\left\{m_{(\sigma)}\right\} \mid,\right. \\
&\left.\left|n_{c}, n_{\bar{c}}, \tilde{m}_{c}, \tilde{m}_{\bar{c}}\right\rangle \longleftrightarrow \mid n_{c}, n_{\bar{c}}\right)\left(m_{c}, m_{\bar{c}} \mid .\right.
\end{aligned}
$$

The inner product in $\hat{\mathcal{V}}$ is given by

$$
\langle A \mid B\rangle=\operatorname{Tr} A^{\dagger} B
$$

Annihilation and creation operators acting on $\hat{\mathcal{V}}$ are defined through

$$
\begin{aligned}
& \left.\left(\begin{array}{c}
a_{(\tau)} \\
a_{(\tau)}^{\dagger}
\end{array}\right)\left|\left\{n_{(\sigma)}\right\},\left\{\tilde{m}_{(\sigma)}\right\}\right\rangle \leftrightarrow\left(\begin{array}{c}
a_{(\tau)} \\
a_{(\tau)}^{\dagger}
\end{array}\right) \mid\left\{n_{(\sigma)}\right\}\right)\left(\left\{m_{(\sigma)}\right\} \mid,\right. \\
& \left.\left(\begin{array}{c}
\tilde{a}_{(\tau)} \\
\tilde{a}_{(\tau)}^{\dagger}
\end{array}\right)\left|\left\{n_{(\sigma)}\right\},\left\{\tilde{m}_{(\sigma)}\right\}\right\rangle \leftrightarrow \mid\left\{n_{(\sigma)}\right\}\right)\left(\left\{m_{(\sigma)}\right\} \mid\left(\begin{array}{c}
a_{(\tau)}^{\dagger} \\
a_{(\tau)}
\end{array}\right),\right.
\end{aligned}
$$


for vector field, and through

$$
\begin{aligned}
& \left.\left(\begin{array}{c}
c \\
\bar{c}
\end{array}\right)\left|n_{c}, n_{\bar{c}}, \tilde{m}_{c}, \tilde{m}_{\bar{c}}\right\rangle \leftrightarrow\left(\begin{array}{c}
c \\
\bar{c}
\end{array}\right) \mid n_{c}, n_{\bar{c}}\right)\left(m_{c}, m_{\bar{c}} \mid,\right. \\
& \left.\left(\begin{array}{c}
c^{\dagger} \\
\bar{c}^{\dagger}
\end{array}\right)\left|n_{c}, n_{\bar{c}}, \tilde{m}_{c}, \tilde{m}_{\bar{c}}\right\rangle \leftrightarrow\left(\begin{array}{c}
c^{\dagger} \\
\bar{c}^{\dagger}
\end{array}\right) \mid n_{c}, n_{\bar{c}}\right)\left(m_{c}, m_{\bar{c}} \mid,\right. \\
& \left.\left(\begin{array}{c}
\tilde{c} \\
\tilde{\bar{c}}
\end{array}\right)\left|n_{c}, n_{\bar{c}}, \tilde{m}_{c}, \tilde{m}_{\bar{c}}\right\rangle \leftrightarrow(-1)^{u+1} \mid n_{c}, n_{\bar{c}}\right)\left(m_{c}, m_{\bar{c}} \mid\left(\begin{array}{c}
c^{\dagger} \\
\bar{c}^{\dagger}
\end{array}\right),\right. \\
& \left.\left(\begin{array}{c}
\tilde{c}^{\dagger} \\
\tilde{\bar{c}}^{\dagger}
\end{array}\right)\left|n_{c}, n_{\bar{c}}, \tilde{m}_{c}, \tilde{m}_{\bar{c}}\right\rangle \leftrightarrow(-1)^{u} \mid n_{c}, n_{\bar{c}}\right)\left(m_{c}, m_{\bar{c}} \mid\left(\begin{array}{c}
c \\
\bar{c}
\end{array}\right),\right.
\end{aligned}
$$

for ghosts, where $u=N_{\text {gh }}\left(n_{c}, n_{\bar{c}}\right)-N_{\text {gh }}\left(m_{c}, m_{\bar{c}}\right)$. Also bases $\left|\left\{n_{(\sigma)}\right\},\left\{\tilde{m}_{(\sigma)}\right\}\right\rangle$ and $\left|n_{c}, n_{\bar{c}}, \tilde{m}_{c}, \tilde{m}_{\bar{c}}\right\rangle$ are generated from the vacuums $|\{0\},\{\tilde{0}\}\rangle$ and $|0,0, \tilde{0}, \tilde{0}\rangle$, respectively, as

$$
\begin{gathered}
\left|\left\{n_{(\sigma)}\right\},\left\{\tilde{m}_{(\sigma)}\right\}\right\rangle=\prod_{\sigma} \frac{\left(a_{(\sigma)}^{\dagger}\right)^{n_{(\sigma)}}\left(\tilde{a}_{(\sigma)}^{\dagger}\right)^{m_{(\sigma)}}}{\sqrt{n_{(\sigma)} ! m_{(\sigma)} !}}|\{0\},\{\tilde{0}\}\rangle, \\
\left|n_{c}, n_{\bar{c}}, \tilde{m}_{c}, \tilde{m}_{\bar{c}}\right\rangle=(-1)^{v}\left(c^{\dagger}\right)^{n_{c}}\left(\bar{c}^{\dagger}\right)^{n_{\bar{c}}}\left(\tilde{c}^{\dagger}\right)^{m_{c}}\left(\tilde{\bar{c}}^{\dagger}\right)^{m_{\bar{c}}}|0,0, \tilde{0}, \tilde{0}\rangle,
\end{gathered}
$$

where $v=m_{c} m_{\bar{c}}$. For the total vacuum, we will use a collective designation $|0, \tilde{0}\rangle$.

Let us introduce thermal vacuums $\langle\theta|$ and $|O(\beta)\rangle \in \hat{\mathcal{V}}$ such that

$$
\langle A\rangle=\langle\theta|A| 0(\beta)\rangle
$$

We require them to satisfy

$$
\left\langle\theta\left|\hat{Q}_{B}^{-}=0, \quad \hat{Q}_{B}^{-}\right| 0(\beta)\right\rangle=0,
$$

and 


$$
\left\langle\theta\left|\hat{Q}_{c}=0, \quad \hat{Q}_{c}\right| O(\beta)\right\rangle=0,
$$

where $\hat{Q}_{B}^{-}$and $\hat{Q}_{c}$ are the generator of the BRS transformation and the ghost hat-charge, respectively, in $\hat{\mathcal{V}}[99]$

$$
\begin{aligned}
& \hat{Q}_{B}^{-}=Q_{B}-\tilde{Q}_{B}=-i\left[a_{(\mathrm{S})}^{\dagger} c-a_{(\mathrm{S})} c^{\dagger}+\tilde{a}_{(\mathrm{S})}^{\dagger} \tilde{c}-\tilde{a}_{(\mathrm{S})} \tilde{c}^{\dagger}\right] \\
& \hat{Q}_{c}=Q_{c}-\tilde{Q}_{c}=c^{\dagger} \bar{c}+\bar{c}^{\dagger} c-\tilde{c}^{\dagger} \overline{\bar{c}}-\tilde{\bar{c}}^{\dagger} \tilde{c} .
\end{aligned}
$$

To satisfy (C.42), we need a trick. Namely, by rewriting (C.26) as

$$
\langle A\rangle=\operatorname{Tr} \theta A \rho \mathrm{e}^{\pi Q_{c}} \theta^{-1}
$$

we introduce an operator $\theta$ with the basic requirement that its inverse exists. Then we settle the correspondence

$$
\langle\theta|\leftrightarrow \theta, \quad| 0(\beta)\rangle \leftrightarrow \rho \mathrm{e}^{\pi Q_{c}} \theta^{-1}
$$

It gives

$$
\begin{aligned}
\langle\theta| \hat{Q}_{c} & =\langle\theta|\left(c^{\dagger} \bar{c}+\bar{c}^{\dagger} c-\tilde{c}^{\dagger} \tilde{\bar{c}}-\tilde{\bar{c}}^{\dagger} \tilde{c}\right) \\
& \leftrightarrow \theta\left(c^{\dagger} \bar{c}+\bar{c}^{\dagger} c\right)-\left(c^{\dagger} \bar{c}+\bar{c}^{\dagger} c\right) \theta \\
& =\left[\theta, Q_{c}\right] .
\end{aligned}
$$

With the requirement given by the first equality in (C.43), the expression in (C.48) is equal to zero and tells us that $\theta$ and $Q_{c}$ commute with each other, i.e. $\left[\theta, Q_{c}\right]=0$. Then

$$
\begin{aligned}
\hat{Q}_{c}|0(\beta)\rangle & =\left(c^{\dagger} \bar{c}+\bar{c}^{\dagger} c-\tilde{c}^{\dagger} \tilde{\bar{c}}^{\tilde{c}}-\tilde{\bar{c}}^{\dagger} \tilde{c}\right)|O(\beta)\rangle \\
& \leftrightarrow\left(c^{\dagger} \bar{c}+\bar{c}^{\dagger} c\right) \rho \mathrm{e}^{\pi Q_{c}} \theta^{-1}-\rho \mathrm{e}^{\pi Q_{c}} \theta^{-1}\left(c^{\dagger} \bar{c}+\bar{c}^{\dagger} c\right) \\
& =\left[Q_{c}, \rho\right] \mathrm{e}^{\pi Q_{c}} \theta^{-1},
\end{aligned}
$$

and the second equality in (C.43) is automatically satisfied as far as $\left[Q_{c}, \rho\right]=0$. Based upon $\left[\theta, Q_{c}\right]=0$ and existence of $\theta^{-1}$, let us try the following form of $\theta$ :

$$
\theta=\mathrm{e}^{i \phi_{1}\left(i c^{\dagger} \bar{c}\right)+i \phi_{2}\left(-i \bar{c}^{\dagger} c\right)+i \phi_{3}\left(i c^{\dagger} \bar{c}\right)\left(-i \bar{c}^{\dagger} c\right)}
$$

where, $\phi_{1}, \phi_{2}$ and $\phi_{3}$ are real numbers which should be determined. Then, taking into account the first correspondence in (C.47), the definition (C.44) and (C.50), the calculation of the first equality in (C.42) goes as 


$$
\begin{aligned}
\langle\theta| \hat{Q}_{B}^{-}= & -i\langle\theta|\left(a_{(\mathrm{S})}^{\dagger} c-a_{(\mathrm{S})} c^{\dagger}+\tilde{a}_{(\mathrm{S})}^{\dagger} \tilde{c}-\tilde{a}_{(\mathrm{S})} \tilde{c}^{\dagger}\right) \\
\leftrightarrow & -i\left\{\theta\left(a_{(\mathrm{S})}^{\dagger} c-a_{(\mathrm{S})} c^{\dagger}\right)+\left(a_{(\mathrm{S})} c^{\dagger}+a_{(\mathrm{S})}^{\dagger} c\right) \theta\right\} \\
= & -i \theta\left\{a_{(\mathrm{S})}^{\dagger} c-a_{(\mathrm{S})} c^{\dagger}+\mathrm{e}^{-i \phi_{1}-i \phi_{3}\left(-i \bar{c}^{\dagger} c\right)} a_{(\mathrm{S})} c^{\dagger}\right. \\
& \left.+\mathrm{e}^{i \phi_{2}+i \phi_{3}\left(i c^{\dagger} \bar{c}\right)} a_{(\mathrm{S})}^{\dagger} c\right\} .
\end{aligned}
$$

If we take $\phi_{1}=0, \phi_{2}=\pi$ and $\phi_{3}=0$, we have $\langle\theta| \hat{Q}_{B}^{-}=0$ with the choice

$$
\theta=\exp \left\{i \pi\left(-i \bar{c}^{\dagger} c\right)\right\}
$$

This structure for $\theta$ allows us to calculate the second equality in (C.42) as

$$
\begin{aligned}
\hat{Q}_{B}^{-}|0(\beta)\rangle & =-i\left(a_{(\mathrm{S})}^{\dagger} c-a_{(\mathrm{S})} c^{\dagger}+\tilde{a}_{(\mathrm{S})}^{\dagger} \tilde{c}-\tilde{a}_{(\mathrm{S})} \tilde{c}^{\dagger}\right)|0(\beta)\rangle \\
& \leftrightarrow-i\left\{\left(a_{(\mathrm{S})}^{\dagger} c-a_{(\mathrm{S})} c^{\dagger}\right) \rho \mathrm{e}^{\pi Q_{c}} \theta^{-1}+\rho \mathrm{e}^{\pi Q_{c}} \theta^{-1}\left(-a_{(\mathrm{S})} c^{\dagger}-a_{(\mathrm{S})}^{\dagger} c\right)\right\} \\
& =Q_{B} \rho \mathrm{e}^{\pi Q_{c}} \theta^{-1}+\rho \mathrm{e}^{\pi Q_{c}} Q_{B} \theta^{-1} \\
& =\left[Q_{B}, \rho\right] \mathrm{e}^{\pi Q_{c}} \theta^{-1},
\end{aligned}
$$

where we also used $\left[\mathrm{e}^{\pi Q_{c}}, Q_{B}\right]_{+}=0$ which is obtained from (C.7). Taking into account the BRS-invariance of the statistical operator $\rho$ (C.27), the expression (C.53) is equal to zero, and both requirements (C.42) are fulfilled with the choice (C.52). We see that introduction of factors $\theta$ and $\theta^{-1}$ indeed is necessary to satisfy the BRS-invariance of the thermal vacuums $\langle\theta|$ and $|0(\beta)\rangle$. Expression of the unit operator in $\mathcal{V}$

$$
\left.\mathbf{1}=\sum_{n, m} \mid n\right) \eta_{n, m}^{-1}(m \mid
$$

and correspondences (C.47) with (C.52) enable us to see the structure of thermal vacuums as

$$
\begin{aligned}
\langle\theta|= & \sum_{n, m}\left(\eta_{n, m}^{-1}\right)^{*}\langle n, \tilde{m}| \theta \\
= & \sum_{n_{(\sigma)}, m_{(\sigma)}}\left(\eta_{n_{(\sigma)}, m_{(\sigma)}}^{-1}\right)^{*}\left\langle\left\{n_{(\sigma)}\right\},\left\{\tilde{m}_{(\sigma)}\right\}\right| \\
& \times \sum_{\substack{n_{c}, n_{\bar{c}} \\
m_{c}, m_{\bar{c}}}}\left(\eta_{\left(n_{c}, n_{\bar{c}}\right),\left(m_{c}, m_{\bar{c})}\right.}^{-1}\right)^{*}\left\langle n_{c}, n_{\bar{c}}, \tilde{m}_{c}, \tilde{m}_{\bar{c}}\right| \theta \\
= & \langle\{0\},\{\tilde{0}\}| \exp \left\{-g^{(\sigma \tau)} \tilde{a}_{(\sigma)} a_{(\tau)}\right\}\langle 0,0, \tilde{0}, \tilde{0}|[1+i \tilde{c} \bar{c}][1+i \tilde{\bar{c}} c] \\
= & \langle 0, \tilde{0}| \exp \left\{i \tilde{c} \bar{c}+i \overline{\bar{c}} c-g^{(\sigma \tau)} \tilde{a}_{(\sigma)} a_{(\tau)}\right\},
\end{aligned}
$$

and 


$$
|0(\beta)\rangle=Z^{-1} \exp \left\{-\mathrm{e}^{-\beta \varepsilon} g^{(\sigma \tau)} a_{(\sigma)}^{\dagger} \tilde{a}_{(\tau)}^{\dagger}-i \mathrm{e}^{-\beta \varepsilon}\left(c^{\dagger} \tilde{c}^{\dagger}+\bar{c}^{\dagger} \tilde{c}^{\dagger}\right)\right\}|0, \tilde{0}\rangle .
$$

It may be instructive to note that considering

$$
\left\langle\theta\left|\hat{Q}_{B}^{+}=0, \quad \hat{Q}_{B}^{+}\right| 0(\beta)\right\rangle=0,
$$

with $\hat{Q}_{B}^{+}=Q_{B}+\tilde{Q}_{B}$, instead of (C.42) with (C.44), leads to the choice $\theta=$ $\exp \left\{i \pi\left(i c^{\dagger} \bar{c}\right)\right\}$.

After determination of parameters $\phi_{i}$ the thermal state conditions with the ghost operators can be derived through the following steps. First, for the bravacuum we see

$$
\begin{gathered}
\langle\theta|\left(\begin{array}{c}
c^{\dagger} \\
\bar{c}^{\dagger}
\end{array}\right) \longleftrightarrow \theta\left(\begin{array}{c}
c^{\dagger} \\
\bar{c}^{\dagger}
\end{array}\right) \\
\langle\theta|\left(\begin{array}{c}
\tilde{c} \\
-\tilde{\bar{c}}
\end{array}\right) \\
\|\left(\begin{array}{c}
c^{\dagger} \\
-\bar{c}^{\dagger}
\end{array}\right) \theta,
\end{gathered}
$$

therefore

$$
\langle\theta|\left(\begin{array}{c}
c^{\dagger} \\
\bar{c}^{\dagger}
\end{array}\right)=\langle\theta|\left(\begin{array}{c}
\tilde{c} \\
-\tilde{\bar{c}}
\end{array}\right) .
$$

Similarly, taking into account structures of the statistical operator $\rho$, the ghost charge $Q_{c},(\mathrm{C} .6)$, and $\theta,(\mathrm{C} .52)$, for the ket-vacuum we have

$$
\begin{gathered}
\left(\begin{array}{c}
c \\
\bar{c}
\end{array}\right)|O(\beta)\rangle \longleftrightarrow\left(\begin{array}{c}
c \\
\bar{c}
\end{array}\right) \rho \mathrm{e}^{\pi Q_{c}} \theta^{-1} \\
\| \\
\left(\begin{array}{r}
\mathrm{e}^{-\beta \varepsilon} \tilde{c}^{\dagger} \\
-\mathrm{e}^{-\beta \varepsilon} \tilde{\bar{c}}^{\dagger}
\end{array}\right)|O(\beta)\rangle \longleftrightarrow \rho \mathrm{e}^{\pi Q_{c}} \theta^{-1}\left(\begin{array}{r}
\mathrm{e}^{-\beta \varepsilon} c \\
-\mathrm{e}^{-\beta \varepsilon} \bar{c}
\end{array}\right),
\end{gathered}
$$

which gives

$$
\left(\begin{array}{c}
c \\
\bar{c}
\end{array}\right)|O(\beta)\rangle=\mathrm{e}^{-\beta \varepsilon}\left(\begin{array}{c}
\tilde{c}^{\dagger} \\
-\tilde{\bar{c}}^{\dagger}
\end{array}\right)|O(\beta)\rangle .
$$


The double tilde conjugation rule must be defined so that it leaves thermal vacuums unchanged. To this end, we put

$$
\begin{aligned}
& \left(\tilde{a}_{(\sigma)}\right)^{\sim}=a_{(\sigma)}, \\
& \left(\begin{array}{c}
\tilde{c} \\
\tilde{\bar{c}}
\end{array}\right)^{\sim}=\left(\begin{array}{l}
\xi c \\
\bar{\xi} \bar{c}
\end{array}\right),
\end{aligned}
$$

and determine parameters $\xi$ and $\bar{\xi}$ so that $\left\langle\left.\theta\right|^{\sim}=\langle\theta| \text { and } \mid O(\beta)\right\rangle^{\sim}=|0(\beta)\rangle$. Taking the tilde conjugation of (C.55) we have

$$
\begin{aligned}
\left\langle\left.\theta\right|^{\sim}\right. & =\left\langle 0,\left.\tilde{0}\right|^{\sim} \exp \left\{-i \xi c \tilde{\bar{c}}-i \bar{\xi} \bar{c} \tilde{c}-g^{(\sigma \tau)} a_{(\sigma)} \tilde{a}_{(\tau)}\right\}\right. \\
& =\langle 0, \tilde{0}| \exp \left\{i \xi \tilde{\bar{c}} c+i \bar{\xi} \tilde{c} \bar{c}-g^{(\sigma \tau)} \tilde{a}_{(\sigma)} a_{(\tau)}\right\}
\end{aligned}
$$

where we assumed $\left\langle 0,\left.\tilde{0}\right|^{\sim}=\langle 0, \tilde{0}|\right.$. The requirement $\left\langle\left.\theta\right|^{\sim}=\langle\theta|\right.$ gives $\xi=1$ and $\bar{\xi}=1$, which leads to

$$
\left(\begin{array}{c}
\tilde{c} \\
\tilde{\bar{c}}
\end{array}\right)^{\sim}=\left(\begin{array}{l}
c \\
\bar{c}
\end{array}\right) .
$$

As a consequence, we obtain the tilde-invariance of the thermal ket-vacuum too:

$$
\begin{aligned}
|0(\beta)\rangle^{\sim} & =\left(Z^{-1} \exp \left\{-\mathrm{e}^{-\beta \varepsilon} g^{(\sigma \tau)} a_{(\sigma)}^{\dagger} \tilde{a}_{(\tau)}^{\dagger}-i \mathrm{e}^{-\beta \varepsilon}\left(c^{\dagger} \tilde{\bar{c}}^{\dagger}+\bar{c}^{\dagger} \tilde{c}^{\dagger}\right)\right\}|0, \tilde{0}\rangle\right)^{\sim} \\
& =Z^{-1} \exp \left\{-\mathrm{e}^{-\beta \varepsilon} g^{(\sigma \tau)} \tilde{a}_{(\sigma)}^{\dagger} a_{(\tau)}^{\dagger}+i \mathrm{e}^{-\beta \varepsilon}\left(\tilde{c}^{\dagger} \bar{c}^{\dagger}+\tilde{\bar{c}}^{\dagger} c^{\dagger}\right)\right\}|0, \tilde{0}\rangle \\
& =|0(\beta)\rangle .
\end{aligned}
$$

A similar line of reasoning can be used to derive the tilde conjugation rule and the thermal state conditions for a system consisting of physical fermions. Let us consider the system specified by the Hamiltonian

$$
H_{\mathrm{f}}=\int d^{3} k \varepsilon(\vec{k}) a^{\dagger}(\vec{k}) a(\vec{k})
$$

with $a(\vec{k})$ and $a^{\dagger}(\vec{k})$ being, respectively, fermion annihilation and creation operators satisfying the canonical anti-commutation relation

$$
\left[a(\vec{k}), a^{\dagger}(\vec{q})\right]_{+}=\delta(\vec{k}-\vec{q})
$$


As in previous consideration, in the following manipulation we will pay attention to one mode, say $\vec{k}$, and drop the index $\vec{k}$ for simplicity. In that case the bases of the state vector space $\mathcal{V}_{\mathrm{f}}$ will be denoted as $\mid 0$ ) and |1) defined by $a \mid 0)=0$ and $\left.\mid 1)=a^{\dagger} \mid 0\right)$.

At the finite temperature, the statistical average of an observable quantity $A$ is given by

$$
\langle A\rangle=\operatorname{Tr} A \rho_{\mathrm{f}}
$$

with $\rho_{\mathrm{f}}$ being the statistical operator of the system

$$
\rho_{\mathrm{f}}=Z_{\mathrm{f}}^{-1} \mathrm{e}^{-\beta H_{\mathrm{f}}}=Z_{\mathrm{f}}^{-1}[\mid 0)\left(0\left|+\mathrm{e}^{-\beta \varepsilon}\right| 1\right)(1 \mid],
$$

where $Z_{\mathrm{f}}$ is the partition function.

Within the doubled state space $\hat{\mathcal{V}}_{\mathrm{f}}=\mathcal{V}_{\mathrm{f}} \otimes \tilde{\mathcal{V}}_{\mathrm{f}}$, the statistical average (C.69) can be expressed in terms of the vacuum expectation with respect to the thermal bra- and ket-vacuums for physical fermions. The bases of $\hat{\mathcal{V}}_{\mathrm{f}}$ are defined through the principle of correspondence:

$$
|n, \tilde{m}\rangle \longleftrightarrow \mid n)(m \mid
$$

where $n$ and $m$ take values of 0 or 1 . Annihilation and creation operators acting on $\hat{\mathcal{V}}_{\mathrm{f}}$ are defined through

$$
\begin{aligned}
\left(\begin{array}{c}
a \\
a^{\dagger}
\end{array}\right)|n, \tilde{m}\rangle & \left.\longleftrightarrow\left(\begin{array}{c}
a \\
a^{\dagger}
\end{array}\right) \mid n\right)(m \mid, \\
\tilde{a}|n, \tilde{m}\rangle & \left.\longleftrightarrow(-1)^{n-m+1} \mid n\right)\left(m \mid a^{\dagger},\right. \\
\tilde{a}^{\dagger}|n, \tilde{m}\rangle & \left.\longleftrightarrow(-1)^{n-m} \mid n\right)(m \mid a .
\end{aligned}
$$

The bases $|n, \tilde{m}\rangle$ are generated from the vacuum $|0, \tilde{0}\rangle$ :

$$
|n, \tilde{m}\rangle=\left(a^{\dagger}\right)^{n}\left(\tilde{a}^{\dagger}\right)^{m}|0, \tilde{0}\rangle
$$

This time we don't have peculiar symmetries for thermal vacuums like in the case of gauge theories with the BRS symmetry. However, in the derivation of thermal vacuums, let us use a trick similar to the one in (C.46)

$$
\langle A\rangle=\operatorname{Tr} \mathrm{e}^{i \phi a^{\dagger} a} A \rho \mathrm{e}^{-i \phi a^{\dagger} a}=\langle\theta|A| O(\beta)\rangle
$$


where $\phi$ is a real number which should be decided. We settle the correspondence for $\langle\theta|$ and $|O(\beta)\rangle$ as

$$
\left\langle\theta\left|\longleftrightarrow \mathrm{e}^{i \phi a^{\dagger} a}, \quad\right| 0(\beta)\right\rangle \longleftrightarrow \rho_{\mathrm{f}} \mathrm{e}^{-i \phi a^{\dagger} a}
$$

They are normalized, $\langle\theta \mid O(\beta)\rangle=1$, and generated from $|0, \tilde{0}\rangle$ as

$$
\begin{aligned}
\langle\theta| & =\langle 0, \tilde{0}|\left[1+\mathrm{e}^{i \phi} \tilde{a} a\right], \\
|0(\beta)\rangle & =Z_{\mathrm{f}}^{-1}\left[1+\mathrm{e}^{-i \phi} \mathrm{e}^{-\beta \varepsilon} a^{\dagger} \tilde{a}^{\dagger}\right]|0, \tilde{0}\rangle .
\end{aligned}
$$

A requirement of the tilde invariance for the thermal bra-vacuum

$$
\left\langle\left.\theta\right|^{\sim}=\langle\theta|\right.
$$

determines the tilde conjugation rule for physical fermion operators up to the phase factor

$$
(a)^{\sim}=\tilde{a}, \quad(\tilde{a})^{\sim}=-\mathrm{e}^{2 i \phi} a .
$$

We have seen in (C.65) that the ghost operators, which are fermion operators, were unchanged under the double tilde conjugation. Let us adopt the same rule for physical fermion operators, i.e. put $\phi=\pi / 2$ to obtain

$$
(a)^{\sim}=\tilde{a}, \quad(\tilde{a})^{\sim}=a .
$$

With this choice of $\phi$, the thermal vacuums for physical fermions read

$$
\begin{aligned}
\langle\theta| & =\langle 0, \tilde{0}|[1+i \tilde{a} a], \\
|0(\beta)\rangle & =Z_{\mathrm{f}}^{-1}\left[1-i \mathrm{e}^{-\beta \varepsilon} a^{\dagger} \tilde{a}^{\dagger}\right]|0, \tilde{0}\rangle
\end{aligned}
$$

and satisfy the following thermal state conditions

$$
\left\langle\theta\left|\tilde{a}^{\dagger}=-i\langle\theta|a, \quad \tilde{a}| O(\beta)\rangle=i \mathrm{e}^{-\beta \varepsilon} a^{\dagger}\right| O(\beta)\right\rangle
$$

\section{Correlation of random force operators}

The random force operators are of the Wiener process whose first and second moments are given by real $c$-numbers: 


$$
\begin{aligned}
\left\langle d F_{t}\right\rangle & =\left\langle d F_{t}^{\dagger}\right\rangle=0, \\
\left\langle d F_{t} d F_{t}\right\rangle & =\left\langle d F_{t}^{\dagger} d F_{t}^{\dagger}\right\rangle=0, \\
\left\langle d F_{t} d F_{t}^{\dagger}\right\rangle & =\text { a real } c \text {-number, } \\
\left\langle d F_{t}^{\dagger} d F_{t}\right\rangle & =\text { a real } c \text {-number, }
\end{aligned}
$$

where $\langle\cdots\rangle=\langle|\cdots|\rangle$ represents the random average referring to the random force operators $d F_{t}$. From (D.1), (D.2) and TSC (6.4) we have for operators (6.14) and (6.15)

$$
\begin{gathered}
\left\langle d W_{t}\right\rangle=\left\langle d \tilde{W}_{t}\right\rangle=\left\langle d W_{t}^{\text {}}\right\rangle=\left\langle d \tilde{W}_{t}^{\text {}}\right\rangle=0, \\
\left\langle d W_{t} d W_{s}\right\rangle=\left\langle d \tilde{W}_{t} d \tilde{W}_{s}\right\rangle=0,
\end{gathered}
$$

while from (6.16) it follows

$$
\begin{aligned}
\left\langle d W_{t}^{q} d W_{s}\right\rangle & =\left\langle d W_{t}^{q} d \tilde{W}_{s}\right\rangle=0, \\
\left\langle d W_{t}^{q} d W_{s}^{q}\right\rangle & =\left\langle d W_{t}^{q} d \tilde{W}_{s}^{q}\right\rangle=0,
\end{aligned}
$$

and their tilde conjugates. Using (D.6) to (D.8) the explicit structure of $d \hat{M}_{t} d \hat{M}_{t}$ in (6.23) is written as

$$
d \hat{M}_{t} d \hat{M}_{t}=-2 \sigma d W_{t} d \tilde{W}_{t} \alpha^{q} \tilde{\alpha}^{q}+\lambda\left(d W_{t} d W_{t}^{q} \alpha^{q} \alpha+d \tilde{W}_{t} d \tilde{W}_{t}^{q} \tilde{\alpha}^{q} \tilde{\alpha}\right)
$$

in a "weak sense" 4 . We demand that the Stratonovich type time evolution generator should not contain a diffusion term, i.e. the term proportional to $\alpha^{q} \tilde{\alpha}^{q}$. Then the correlation $\left\langle d W_{t} d \tilde{W}_{t}\right\rangle$ is determined to be

$$
d W_{t} d \tilde{W}_{t}=\tau\{2 \kappa(t)[n(t)+\eta]+\dot{n}(t)\} d t=\left\langle d W_{t} d \tilde{W}_{t}\right\rangle
$$

so that $\hat{\Pi}_{\mathrm{D}}$ in (6.23) is cancelled by the first term in the r.h.s. of (D.9). Here, the first equality in (D.10) should be understood in a weak sense as well. Expression (D.10) is compatible with the assumption that the process is white. Let us put the subscript $F$ to $\Sigma^{<}(t)$ in the Boltzmann equation in order to remember that it is due to the interaction with the random force $d F_{t}$ :

$$
\dot{n}(t)=-2 \kappa(t) n(t)+i \Sigma_{F}^{<}(t) .
$$

Making use of two previous equations, we have

$\overline{4}$ In the case of classical systems it corresponds to the stochastic convergence. 


$$
\begin{aligned}
i \Sigma_{F}^{<}(t) d t & =2 \kappa(t) n(t) d t+\dot{n}(t) d t \\
& =-2 \kappa(t) \eta d t+\sigma \tau\left\langle d W_{t} d \tilde{W}_{t}\right\rangle \\
& =-2 \kappa(t) \eta d t+\left\langle d F_{t}^{\dagger} d F_{t}\right\rangle+\nu\left[\left\langle d F_{t} d F_{t}^{\dagger}\right\rangle-\sigma\left\langle d F_{t}^{\dagger} d F_{t}\right\rangle\right]
\end{aligned}
$$

where (6.14) has been used, and $\mu$ has been erased with the help of (5.32).

We can assume that the quantity $\eta$ may depend on $\nu$, i.e. $\eta=\eta(\nu)$, and that the physical quantities $\kappa(t), \Sigma_{F}^{<}(t),\left\langle d F_{t}^{\dagger} d F_{t}\right\rangle$, and $\left\langle d F_{t} d F_{t}^{\dagger}\right\rangle$ may not depend on $\nu$. Then, differentiating equation (D.12) with respect to $\nu$, one has

$$
0=-2 \kappa(t) \frac{\partial \eta}{\partial \nu} d t+\left\langle d F_{t} d F_{t}^{\dagger}\right\rangle-\sigma\left\langle d F_{t}^{\dagger} d F_{t}\right\rangle
$$

This leads to

$$
\frac{\partial \eta}{\partial \nu}=k(t)
$$

which is solved as

$$
\eta=k(t) \nu+l(t)
$$

where $k(t)$ and $l(t)$ are real numbers independent of $\nu$. With this solution one has

$$
\left\langle d F_{t} d F_{t}^{\dagger}\right\rangle-\sigma\left\langle d F_{t}^{\dagger} d F_{t}\right\rangle=2 \kappa(t) k(t) d t,
$$

and

$$
i \Sigma_{F}^{<}(t) d t=-2 \kappa(t) l(t) d t+\left\langle d F_{t}^{\dagger} d F_{t}\right\rangle,
$$

which leads to

$$
\left\langle d F_{t}^{\dagger} d F_{t}\right\rangle=\{2 \kappa(t)[l(t)+n(t)]+\dot{n}(t)\} d t,
$$

where we have used (D.11). The substitution of (D.18) into (D.16) gives us

$$
\left\langle d F_{t} d F_{t}^{\dagger}\right\rangle=\{2 \kappa(t)[k(t)+\sigma l(t)+\sigma n(t)]+\sigma \dot{n}(t)\} d t .
$$

For the case of stationary quantum stochastic process, the Boltzmann equation (D.11) reduces to 


$$
\dot{n}(t)=-2 \kappa[n(t)-\bar{n}]
$$

where $\bar{n}$ is the average quantum number in equilibrium. Therefore, (D.18) and (D.19) reduce, respectively, to

$$
\begin{aligned}
\left\langle d F_{t}^{\dagger} d F_{t}\right\rangle & =2 \kappa[\bar{n}+l(t)] d t \\
\left\langle d F_{t} d F_{t}^{\dagger}\right\rangle & =2 \kappa[k(t)+\sigma l(t)+\sigma \bar{n}] d t
\end{aligned}
$$

Since in the white noise assumption the Boltzmann equation (D.20) is compatible with the stationary process specified by [41]

$$
\begin{aligned}
& \left\langle d F_{t}^{\dagger} d F_{t}\right\rangle=2 \kappa \bar{n} d t \\
& \left\langle d F_{t} d F_{t}^{\dagger}\right\rangle=2 \kappa[1+\sigma \bar{n}] d t
\end{aligned}
$$

one concludes now that

$$
l(t)=0, \quad k(t)=1,
$$

which leads to

$$
\eta=\nu, \quad \xi=\mu .
$$

Note that the result (D.24) can be obtained using the Bogoliubov transformation as it is described in Appendix B.

Substituting (D.25) into (D.18) and (D.19), one obtains

$$
\begin{aligned}
& \left\langle d F_{t}^{\dagger} d F_{t}\right\rangle=[2 \kappa(t) n(t)+\dot{n}(t)] d t \\
& \left\langle d F_{t} d F_{t}^{\dagger}\right\rangle=\{2 \kappa(t)[1+\sigma n(t)]+\sigma \dot{n}(t)\} d t
\end{aligned}
$$

which leads to

$$
\left\langle d W_{t} d W_{t}^{\text {q }}\right\rangle=\left\langle d F_{t}, d F_{t}^{\dagger}\right\rangle-\sigma\left\langle d F_{t}^{\dagger}, d F_{t}\right\rangle=2 \kappa(t) d t .
$$

Assembling (D.9), (D.10), (D.26) and (D.29) one obtains expression (6.25).

\section{References}

[1] T. Arimitsu, Cond. Matt. Phys. Issue 4, 26 (1994).

Accessible at http://www.px.tsukuba.ac.jp/home/tcm /arimitsu/cmp4.pdf]. 
[2] T. Arimitsu and H. Umezawa, Progr. Theor. Phys. 74, 429 (1985).

[3] T. Arimitsu and H. Umezawa, Progr. Theor. Phys. 77, 32 (1987).

[4] T. Arimitsu and H. Umezawa, Progr. Theor. Phys. 77, 53 (1987).

[5] N.N. Bogoliubov, In series: Studies in Statistical Mechanics, vol. 1, p. 1-118. Ed. by J. de Boer and G.E. Uhlenbeck (North-Holland, Amsterdam, 1962).

[6] S. Nakajima, Progr. Theor. Phys. 20, 948 (1958).

[7] R. Zwanzig, J. Chem. Phys. 33, 1338 (1960).

[8] R. Zwanzig, In series: Lectures in Theoretical Physics, vol. III, p. 106-141. Ed. by W.E. Brittin, B.W. Downs and J. Downs (Interscience Publishers, Inc., New York, 1961).

[9] H.-P. Breuer and F. Petruccione, The Theory of Open Quantum Systems (Oxford University Press, Oxford, 2002).

[10] I. Prigogine, C. George and F. Henin, Physica A 45, 418 (1969).

[11] I. Prigogine, Physica A 263, 528 (1999).

[12] H. Mori, Progr. Theor. Phys. 33, 423 (1965).

[13] K. Kawasaki and J.D. Gunton, Phys. Rev. A 8, 2048 (1973).

[14] B. Robertson, Phys. Rev. 144, 151 (1966).

[15] M.S. Green, J. Chem. Phys. 20, 1281 (1952).

[16] M.S. Green, J. Chem. Phys. 22, 398 (1954).

[17] R. Kubo, J. Phys. Soc. Japan 12, 570 (1957).

[18] R. Kubo, M. Yokota and S. Nakajima, J. Phys. Soc. Japan 12, 1203 (1957).

[19] D.N. Zubarev, Nonequilibrium Statistical Thermodynamics (Consultant Bureau, New York, 1974).

[20] D.N. Zubarev, J. Soviet Mathem. 16, 1509 (1981).

[21] S. Chandrasekhar, Rev. Mod. Phys. 15, 1 (1943).

[22] M.C. Wang and G.E. Uhlenbeck, Rev. Mod. Phys. 17, 323 (1945).

[23] C.W. Gardiner, Handbook of Stochastic Methods for Physics, Chemistry and Natural Sciences, 2nd edn. (Springer-Verlag, Berlin, 1985).

[24] K. Itô, Proc. Imp. Acad. Tokyo 20, 519 (1944).

[25] R. Stratonovich, J. SIAM Control 4, 362 (1966).

[26] Stochastic Processes in Chemical Physics. Edited by K.E. Shuler. In series: Advances in Chemical Physics, vol. XV. Ed. by I. Prigogine and S. Rice (Interscience Publishers, New York, 1969). 
[27] W. Coffey, In: Dynamical Processes in Condensed Matter, p. 69-252. Ed. by M.W. Evans. In series: Advances in Chemical Physics, vol. LXIII. Ed. by I. Prigogine and S. Rice (Interscience Publishers, New York, 1985).

[28] K. Sobczyk, Stochastic Differential Equations: with Application to Physics and Engineering (Kluwer Academic, Dordrecht, 1991).

[29] N.G. van Kampen, Stochastic Processes in Physics and Chemistry (North Holland, Amsterdam, 1992).

[30] W.T. Coffey, Yu.P. Kalmykov and J.T. Waldron, The Langevin Equation. With Applications in Physics, Chemistry and Electrical Engineering (World Scientific, Singapore, 1996).

[31] P.W. Anderson, J. Phys. Soc. Japan 9, 316 (1954).

[32] R. Kubo, J. Phys. Soc. Japan 9, 935 (1954).

[33] R. Kubo, In: Fluctuation, Relaxation and Resonance in Magnetic Systems, p. 23-68. Ed. by D. ter Haar (Oliver and Boyd, Edinburgh-London, 1962).

[34] R. Kubo, J. Math. Phys. 4, 174 (1963).

[35] I.R. Senitzky, Phys. Rev. 119, 670 (1960).

[36] I.R. Senitzky, Phys. Rev. 124, 642 (1961).

[37] I.R. Senitzky, Phys. Rev. 131, 2827 (1963).

[38] J. Schwinger, J. Math. Phys. 2, 407 (1961).

[39] H. Haken, Z. Phys. 181, 96 (1964).

[40] H. Haken, Z. Phys. 182, 346 (1965).

[41] H. Haken, Optik. Handbuch der Physik, vol. XXV/2c (Berlin, Springer-Verlag, 1970). Reprinted as H. Haken, Laser Theory (Berlin, Springer-Verlag, 1984).

[42] H. Haken, Rev. Mod. Phys. 47, 67 (1975).

[43] M. Lax, Phys. Rev. 145, 110 (1966).

[44] R. Kubo, J. Phys. Soc. Japan Suppl. 26, 1 (1969).

[45] E.B. Davies, Commun. Math. Phys. 15, 277 (1969).

[46] E.B. Davies, Quantum Theory of Open Systems (Academic Press, London, 1976).

[47] A.M. Cockroft and R.L. Hudson, J. Multivar. Anal. 7, 107 (1977).

[48] R.L. Hudson and R.F. Streater, Phys. Lett. A 86, 277 (1981).

[49] R.L. Hudson and K.R. Parthasarathy, Commun. Math. Phys. 93, 301 (1984).

[50] R.L. Hudson and K.R. Parthasarathy, Acta Appl. Math. 2, 353 (1984). 
[51] R.L. Hudson and J.M. Lindsay, In: Quantum Probability and Applications II, p. 276-305. In series: Lecture Notes in Mathematics, vol. 1136. Ed. by L. Accardi and W. von Waldenfels (Berlin, Springer, 1984).

[52] R.L. Hudson and J.M. Lindsay, J. Func. Anal. 61, 202 (1985).

[53] L. Accardi, A. Frigerio and J.T. Lewis, Publ. RIMS, Kyoto Univ. 18, 97 (1982).

[54] L. Accardi, Rev. Math. Phys. 2, 127 (1990).

[55] K.R. Parthasarathy, Pramãna - J. Phys. 25, 457 (1985).

[56] K.R. Parthasarathy, Rev. Math. Phys. 1, 89 (1989).

[57] K.R. Parthasarathy, An Introduction to Quantum Stochastic Calculus. In series: Monographs in Mathematics, vol. 85 (Basel, Birkhäuser, 1992).

[58] D. Applebaum and R.L. Hudson, Commun. Math. Phys. 96, 473 (1984).

[59] D. Applebaum and R.L. Hudson, J. Math. Phys. 25, 858 (1984).

[60] D. Applebaum, J. Phys. A 19, 937 (1986).

[61] D. Applebaum, J. Phys. A 28, 257 (1995).

[62] R.L. Hudson and K.R. Parthasarathy, Commun. Math. Phys. 104, 457 (1986).

[63] K.R. Parthasarathy and K.B. Sinha, Pramãna - J. Phys. 27, 105 (1986).

[64] C. Barnett, R.F. Streater and I.F. Wilde, J. Funct. Anal. 48, 172 (1982).

[65] C. Barnett, R.F. Streater and I.F. Wilde, J. London Math. Soc. 27, 373 (1983).

[66] C. Barnett, R.F. Streater and I.F. Wilde, Commun. Math. Phys. 89, 13 (1983).

[67] C. Barnett, R.F. Streater and I.F. Wilde, J. Func. Anal. 52, 19 (1983).

[68] T. Arimitsu, Phys. Lett. A 153, 163 (1991).

[69] T. Arimitsu, M. Ban and T. Saito, Physica A 177, 329 (1991).

[70] T. Saito and T. Arimitsu, Mod. Phys. Lett. B 6, 1319 (1992).

[71] T. Saito and T. Arimitsu, Mod. Phys. Lett. B 7, 623 (1993).

[72] T. Saito and T. Arimitsu, Mod. Phys. Lett. B 7, 1951 (1993).

[73] T. Saito and T. Arimitsu, J. Phys. A 30, 7573 (1997).

[74] T. Arimitsu, Phys. Essays 9, 591 (1996).

[75] T. Arimitsu, In: Non-Commutativity, Infinite-Dimensionality and Probability at the Crossroads, p. ???-???. Ed. by L. Accardi and S. Tasaki. In series: Quantum Probability and White Noise Analysis, vol. 17 (World Scientific, Singapore, 2003).

[76] T. Arimitsu, J. Pradko and H. Umezawa, Physica A 135, 487 (1986). 
[77] C.W. Gardiner and M.J. Collet, Phys. Rev. A 31, 3761 (1985).

[78] C.W. Gardiner and P. Zoller, Quantum Noise: A Handbook of Markovian and non-Markovian Quantum Stochastic Methods with Applications to Quantum Optics, 2nd edn. In: Springer Series in Synergetics, vol. 56 (Springer, Berlin, 2000).

[79] T. Arimitsu, Y. Sudo and H. Umezawa, Physica A 146, 433 (1987).

[80] T. Arimitsu, M. Guida and H. Umezawa, Europhys. Lett. 3, 277 (1987).

[81] T. Arimitsu, M. Guida and H. Umezawa, Physica A 148, 1 (1988).

[82] T. Arimitsu and H. Umezawa, In: Advances on Phase Transitions and Disordered Phenomena. Ed. by G. Busiello, L. De Cesare, F. Mancini and M. Marinaro (World Scientific, Singapore, 1987) p. 483-504.

[83] H. Umezawa and T. Arimitsu, In: Foundation of Quantum Mechanics - In the Light of New Technology. Ed. by M. Namiki, Y. Ohnuki, Y. Murayama and S. Nomura (Physical Society of Japan, Tokyo, 1987) p. 79-90.

[84] T. Arimitsu, H. Umezawa, Y. Yamanaka and P. Papastamatiou, Physica A 148, 27 (1988).

[85] R. Kubo, H. Ichimura, T. Usui and N. Hashitsume, Statistical Mechanics. An Advanced Course with Problems and Solutions (North-Holland, Amsterdam, 1965).

[86] R. Balescu, Equilibrium and Non-Equilibrium Statistical Mechanics (Wiley Interscience, New York, 1975).

[87] J. Dalibard, Y. Castin and K. Mølmer, Phys. Rev. Lett. 68, 580 (1992).

[88] K. Mølmer, Y. Castin and J. Dalibard, J. Opt. Soc. Am. B 10, 524 (1993).

[89] K. Mølmer and Y. Castin, Quant. and Semiclass. Opt. 8, 49 (1996).

[90] C.W. Gardiner, A.S. Parkins and P. Zoller, Phys. Rev. A 46, 4363 (1992).

[91] R. Dum, A.S. Parkins, P.Zoller and C.W. Gardiner, Phys. Rev. A 46, 4382 (1992).

[92] H. J. Carmichael, An Open Systems Approach to Quantum Optics. In series: Lecture Notes in Physics, vol. m18 (Springer-Verlag, Berlin 1993).

[93] B.M. Garraway and P.L. Knight, Phys. Rev. A 49, 1266 (1994).

[94] M.B. Plenio and P.L. Knight, Rev. Mod. Phys. 70, 101 (1998).

[95] T. Arimitsu, In: Stochastic Processes and their Applications, p. 279-294. Ed. by A. Vijayakumar and M. Sreenivasan (Narosa Publ. House, New Dehli, 1999).

[96] T. Arimitsu and Y. Kaburaki, private communication. 
[97] T. Saito and T. Arimitsu, In: Stochastic Processes and their Applications, p. 323-333. Ed. by A. Vijayakumar and M. Sreenivasan (Narosa Publ. House, New Dehli, 1999).

[98] T. Arimitsu, In: Non-Commutativity, Infinite-Dimensionality and Probability at the Crossroads, p. 206-224. Ed. by N. Obata, T. Matsui and A. Hora. In series: Quantum Probability and White Noise Analysis, vol. 16 (World Scientific, Singapore, 2003).

[99] I. Ojima, Ann. Phys. (NY) 137, 1 (1981).

[100] T. Hayashi, Master Thesis. University of Tsukuba, Japan, 2002 (in Japanese).

[101] L.D. Faddeev, V. Popov, Phys. Lett. B 25, 29 (1967).

[102] C. Becchi, A. Rouet and R. Stora, Ann. Phys. (NY) 98, 287 (1976).

[103] T. Kugo and I. Ojima, Phys. Lett. B 73, 459 (1978).

[104] T. Kugo and I. Ojima, Progr. Theor. Phys. Suppl. 66, 1 (1979).

[105] H. Hata and T. Kugo, Phys. Rev. D 21, 3333 (1980). 